\title{
Method of integral equations in the polytropic theory of stars with axial rotation. I. Polytropes $n=0$ and $n=1$
}

\author{
Vavrukh M. V., Dzikovskyi D. V. \\ Ivan Franko National University of Lviv, \\ 8 Kyrylo and Methodiy Str., 79005, Lviv, Ukraine
}

(Received 7 November 2020; Accepted 19 April 2021)

\begin{abstract}
Calculations of characteristics of stars with axial rotation in the frame of polytropic model are based on the solution of mechanical equilibrium equation - differential equation of second order in partial derivatives. Different variants of approximate determinations of integration constants are based on traditional in the theory of stellar surface approximation, namely continuity of gravitational potential in the surface vicinity. We proposed a new approach, in which we used simultaneously differential and integral forms of equilibrium equations. This is a closed system and allows us to define in self-consistent way integration constants, the polytrope surface shape and distribution of matter over volume of a star. With the examples of polytropes $n=0$ and $n=1$, we established the existence of two rotation modes (with small and large eccentricities). It is proved that the polytrope surface is the surface of homogeneous rotational ellipsoid for the case $n=0$. The polytrope characteristics with $n=1$ in different approximations were calculated as the functions of angular velocity. For the first time it has been calculated the deviation of polytrope surface at fixed value of angular velocity from the surface of associated rotational ellipsoid.
\end{abstract}

Keywords: polytropic stars, heterogeneous ellipsoids, axial rotation, mechanical equilibrium equation, stability of stars.

2010 MSC: $85-08,85 \mathrm{~A} 15$

DOI: $10.23939 / \mathrm{mmc} 2021.02 .338$

\section{Introduction}

Axial rotation is a factor which is inherent to all stars of different types, as well as to the majority of celestial bodies and their systems. It is well known that normal stars of early spectral classes are characterized by high angular velocity, and stars of late classes (older than F5) have a small angular velocity and are surrounded by planets. For example, the stars of classes B $\div \div \mathrm{F} 0$ have angular velocity which exceeds $10^{-5} \mathrm{~s}^{-1}$ [1], while the angular velocity of the Sun is $3 \cdot 10^{-6} \mathrm{~s}^{-1}$. The angular velocity of neutron stars (pulsars) are $10 \mathrm{~s}^{-1} \leqslant \omega \leqslant 4 \cdot 10^{3} \mathrm{~s}^{-1}[2]$.

The fundamentals of the polytropic model of stars in the frame of model without axial rotation were developed in the following works: Lane [3], Emden [4], Fowler [5], Eddington [6] and others. In the fundamental work of Milne [7], authors for the first time studied the influence of axial rotation of stars on their characteristics - density distribution, temperature, and mass, within the frame of Eddington model which corresponds to the polytropic model with the index $n=3$. In ten years, Chandrasekhar used the Milne method and received approximate numerical solutions of mechanical equilibrium of stars within the frame of polytropic model for indices $n=1.0,1.5,2.0,3.0,4.0$ and calculated dependence of geometrical characteristics, mass, volume on the angular velocity for the case of small velocities. The results obtained by him correspond to the perturbation theory of the first order relative to the squared angular velocity [8]. In this work, in essence, a pure mathematical problem was solved and that became a traditional approach for later works, which were performed over last century. In the later work of James [9], the author calculated numerically equatorial and polar radii, mass and moment of inertia relative to the axis rotation for the polytrope models in much wider range of angular velocities. For a long time, the results received by him were considered as standards. However, this publication does 
not contain any data about solutions of equilibrium equation, on the basis of which it would be possible to calculate other characteristics of rotational polytrope. Kopal [10] noted that in the particular case $n=1$ the equilibrium equation is a linear differential equation and its angular and radial variables are separable, therefore its solutions are the products of spherical Bessel functions on the Legendre polynomials. However, Kopal has not found the general solution which corresponds to the boundary conditions and requires the calculation of the set of integration constants. Such solution was found in the work of Williams [11] in the form of expansion and including the polynomial $P_{8}(\cos \theta)$. In the work [12], the author generalized the Milne approach by more detailed description of the peripheral region and used fitting parameters for merging the solutions at the boundary between inner region and periphery. The obtained approximations for solutions are linear relative to the squared angular velocity, and fitting parameters and integration constant do not depend on angular velocity.

General approximation which is used in the works $[8,11,12]$ for finding of integration constants are traditional approximation in the theory of stellar surface. It assumes that the gravitational potential in periphery region is formed by distribution of matter in inner region and has a standard multipole form. It is considered that the influence of matter distribution in periphery region on the formation of gravitational potential inside periphery is negligibly small due to the small matter density. The gravitational potential in inner region determines the solution of mechanical equilibrium equation. Continuity condition of potential on the separation surface allows us to find integration constants. With this purpose, in the work [8] the author choses the separation surface in the form of sphere with Emden radius. The sphere with the radius smaller than the Emden radius was used in the work [12] to find the integration constant and fitting parameters. In the work [11], the separtion surface was chosen in the form of rotational ellipsoid, that yielded opportunity to find integration constants (at $n=1$ ) as functions of angular velocity.

In the work [13], the integration constant for the case $n=0$ was found in self-consistent way assuming that the surface of rotational polytrope is the surface of rotational ellipsoid. This method improves slightly the Chandrasekhar solution for $n=1$ (in the region of small velocities).

The results obtained in the work [9] for the polar and equatorial radii, as well as critical angular velocity, at which instability occurs, played role of standards for approximate calculations performed in the works $[11,12]$.

A new method for finding solutions of the differential stellar equilibrium equation with axial rotation in the polytropic model was proposed in our works [14-16]. We represent the solutions in the form of multicomponent expansions, and the set of integration constants we determine using the integral form of equilibrium equation. As a result, the set of integration constants is determined by a system of linear algebraic equations, and integration constants are functions of angular velocity. In the works [14-16], we used a self-consistent approach assuming that the polytrope surface is the surface of rotational ellipsoid with two parameters (equatorial radius and eccentricity) calculated numerically by the iterative procedure.

Inspite of the century-long history of a study of stars in the frame of polytropic model, this approach still has its methodological and applied meaning. The polytropic model is a good zero approximation in the theory of white dwarfs $[17,18]$, in the theory of main sequence stars [19,20], circumstellar disks, giant planets, neutron stars and black holes [21]. In the works performed in the XXI century, the main focus is not on methodological details of finding the solutions of equilibrium equation as a differential equation of second order in partial derivatives, but the applied aspects are mostly considered. As an example of research area is a search for parameters of polytropic equation of state for specific observed stars with high angular velocities, that is, the construction of polytropic models of stars with known observable characteristics. However, the preassigned index value $n=1$ (as in the works [19, 20]) of polytropic models does mean restriction, which reduces the value of such approach.

In present work we represent an improved approach developed in the works [14-16]. Here we generalize the self-consistent approach (the method of trial functions) and specify the system of linear equations for integration constants and demonstrate advantages of the use of the integral form of equilibrium equation. The geometrical characteristics of rotational polytropes, mass, and moment of inertia have been calculated as the functions of angular velocity. 


\section{General relations}

The single-phase model of a star is generally accepted in the polytropic theory. It is based on the polytropic equation of state

$$
P(\mathbf{r})=K \rho^{\nu}(\mathbf{r})=K \rho^{1+1 / n}(\mathbf{r}),
$$

where $P(\mathbf{r})$ is the pressure at point with radius-vector $\mathbf{r}, \rho(\mathbf{r})$ is the local density in this point, and constants $K, n$ are the parameters of the model.

Using non-inertial reference system, with the presence of rotation the equilibrium equation of model is written in the form [22]

$$
\nabla P(\mathbf{r})=-\rho(\mathbf{r})\left\{\nabla \Phi_{\text {grav }}(\mathbf{r})+\nabla \Phi_{\mathrm{c}}(\mathbf{r})\right\},
$$

where

$$
\Phi_{\text {grav }}(\mathbf{r})=-G \int \frac{\rho\left(\mathbf{r}^{\prime}\right) d \mathbf{r}^{\prime}}{\left|\mathbf{r}-\mathbf{r}^{\prime}\right|}
$$

is the gravitational potential inside a star, and $\Phi_{\mathrm{c}}(\mathbf{r})$ is the centrifugal potential. Let the axis $O z$ of spherical coordinate system coincides with the axis of rotation then

$$
\Phi_{\mathrm{c}}(\mathbf{r})=-\frac{1}{2} \omega^{2} r^{2} \sin ^{2} \theta
$$

Here $\theta$ is the polar angle, $\omega$ is the angular velocity of the reference frame, which is considered to be constant. Substituting expressions (1), (3) and (4) in equation (2) and taking into account the identity

$$
\left(1+\frac{1}{n}\right) \rho^{1 / n-1}(\mathbf{r}) \nabla \rho(\mathbf{r})=(1+n) \nabla \rho^{1 / n}(\mathbf{r}),
$$

we get the equilibrium equation in the form of differential equation

$$
K(1+n) \Delta \rho^{1 / n}(\mathbf{r})=-4 \pi G \rho(\mathbf{r})+\frac{1}{2} \omega^{2} \Delta\left(r^{2} \sin ^{2} \theta\right),
$$

which determines the density distribution. In the presence of axial symmetry and symmetry relative to the equatorial plane in the density distribution $(\rho(\mathbf{r})=\rho(r, \theta)=\rho(r, \pi-\theta))$ the Laplace operator is written in the form

$$
\Delta=\Delta_{r}+\frac{1}{r^{2}} \Delta_{\theta}, \quad \Delta_{r}=\frac{1}{r^{2}} \cdot \frac{\partial}{\partial r}\left(r^{2} \frac{\partial}{\partial r}\right), \quad \Delta_{\theta}=\frac{\partial}{\partial t}\left(1-t^{2}\right) \frac{\partial}{\partial t},
$$

where $t=\cos \theta$, moreover $\Delta\left(r^{2} \sin ^{2} \theta\right)=4$. Let us introduce dimensionless variables

$$
\xi=r / \lambda_{n}, \quad Y_{n}(\xi, \theta)=\left[\rho(r, \theta) / \rho_{\mathrm{c}}\right]^{1 / n},
$$

where $\rho_{\mathrm{c}}$ is the matter density in stellar center. In the dimensionless form equation (6) takes the form

$$
\Delta_{\xi, \theta} Y_{n}(\xi, \theta)=\Omega^{2}-Y_{n}^{n}(\xi, \theta),
$$

if the scale of length $\lambda_{n}$ and the dimensionless angular velocity $\Omega$ are determined by relations

$$
K(1+n)=4 \pi G \lambda_{n}^{2} \rho_{\mathrm{c}}^{1-1 / n}, \quad \Omega=\omega\left(2 \pi G \rho_{\mathrm{c}}\right)^{-1 / 2},
$$

and

$$
\Delta_{\xi, \theta}=\Delta_{\xi}+\frac{1}{\xi^{2}} \Delta_{\theta}, \quad \Delta_{\xi}=\frac{1}{\xi^{2}} \cdot \frac{\partial}{\partial \xi}\left(\xi^{2} \frac{\partial}{\partial \xi}\right)
$$

According to the definition (8) $Y_{n}(0, \theta)=1$, the condition $\partial Y_{n}(\xi, \theta) / \partial \xi=0$ at $\xi=0$ corresponds to the solutions regularly in the vicinity $\xi=0$. The important conclusion follows from these boundary 
conditions: the asymptotics of the equation solutions (9) at $\xi \ll 1$ and fixed value $\Omega$ do not depend on the polytropic index

$$
Y_{n}(\xi, \theta)=Y_{0}(\xi, \theta) .
$$

In the case of large values $\Omega$, it is possible a non-monotonic dependence $Y_{n}(\xi, \theta)$ on the variable $\xi$ in the equator vicinity, which leads to leakage of matter. The conditions of stellar stability in the equator vicinity

$$
Y_{n}\left(\xi, \frac{\pi}{2}\right)=0, \quad \frac{\partial}{\partial \xi} Y_{n}\left(\xi, \frac{\pi}{2}\right)=0
$$

determines the maximum value of the parameter $\Omega_{\max }(n)$ and its corresponding value of the equatorial radius $\xi_{e}^{\max }(n)$. It is obviously that at $\Omega<\Omega_{\max }(n)$ instead of conditions (13) the following conditions are satisfied

$$
Y_{n}\left(\xi, \frac{\pi}{2}\right)=0, \quad \frac{\partial}{\partial \xi} Y_{n}\left(\xi, \frac{\pi}{2}\right)<0 .
$$

According to the definition (8), the physical meaning has only positive solutions of the equation (9), which is two-dimensional differential equation of second order in partial derivatives with two dimensionless parameters $n, \Omega \geqslant 0$.

The equation (9) can be considered as a equation for dimensionless gravitational potential, created by the distribution of the dimensionless matter density $(4 \pi)^{-1}\left\{\Omega^{2}-Y_{n}^{n}(\xi, \theta)\right\}$. In this regard, it can be rewritten in integral form

$$
Y_{n}(\xi, \theta)=1+\sum_{i=1}^{\infty} C_{2 l} \xi^{2 l} P_{2 l}(t)-\frac{1}{4 \pi} \int\left\{\Omega^{2}-Y_{n}^{n}\left(\xi^{\prime}, \theta^{\prime}\right)\right\} Q\left(\boldsymbol{\xi}, \boldsymbol{\xi}^{\prime}\right) d \boldsymbol{\xi}^{\prime},
$$

where $C_{2 l}$ are integration constants, $P_{2 l}(t)$ are the Legendre polynomials of $2 l$-th order, the kernel of the equation is

$$
Q\left(\boldsymbol{\xi}, \boldsymbol{\xi}^{\prime}\right)=\left|\boldsymbol{\xi}-\boldsymbol{\xi}^{\prime}\right|^{-1}-\left(\xi^{\prime}\right)^{-1},
$$

and the integration is performed over the stellar volume. Taking into account the identity

$$
\Delta_{\xi, \theta}\left\{\xi^{n} P_{n}(t)\right\}=0,
$$

we see, that equations (9) and (15) are equivalent.

The gravitational potential inside a star (3) is related to the dimensionless potential

$$
\Phi_{n}(\boldsymbol{\xi})=\Phi_{n}(\xi, \theta)=-\frac{1}{4 \pi} \int \frac{Y_{n}^{n}\left(\xi^{\prime}, \theta^{\prime}\right)}{\left|\boldsymbol{\xi}-\boldsymbol{\xi}^{\prime}\right|} d \boldsymbol{\xi}^{\prime}
$$

by expression

$$
\Phi_{\text {grav }}(\mathbf{r})=4 \pi G \rho_{c} \lambda_{n}^{2} \Phi_{n}(\boldsymbol{\xi}) .
$$

Therefore, equation (15) can be rewritten in terms $Y_{n}(\xi, \theta)$ and $\Phi_{n}(\xi, \theta)$, namely

$$
Y_{n}(\xi, \theta)+\left\{\Phi_{n}(\xi, \theta)-\Phi_{n}(0,0)\right\}=1+\sum_{l=1}^{\infty} C_{2 l} \xi^{2 l} P_{2 l}(t)+\Omega^{2}\left\{\Phi_{0}(\xi, \theta)-\Phi_{0}(0,0)\right\},
$$

where $\Phi_{0}(\xi, \theta)$ is determined by expression (18) at $Y_{n}\left(\xi^{\prime}, \theta^{\prime}\right) \equiv 1$. From the equation (2), which is rewritten in the dimensionless form follows the relation

$$
\frac{\partial}{\partial \xi}\left\{\Phi_{n}(\xi, \theta)+Y_{n}(\xi, \theta)\right\}=\frac{\Omega^{2}}{3} \xi\left\{1-P_{2}(t)\right\} .
$$

Using equality (20), equality (21) can be rewritten in such form [7]:

$$
\frac{\partial}{\partial \xi}\left\{\sum_{i=1}^{\infty} C_{2 l} \xi^{2 l} P_{2 l}(t)+\Omega^{2}\left[\Phi_{0}(\xi, \theta)-\Phi_{0}(0,0)\right]\right\}=\xi \frac{\Omega^{2}}{3}\left(1-P_{2}(t)\right) .
$$


The difference of potentials $\Phi_{0}(\xi, \theta)-\Phi_{0}(0,0)$ are calculated by integration over volume of the polytrope with index $n$. Using expansion in the series of kernel $Q\left(\boldsymbol{\xi}, \boldsymbol{\xi}^{\prime}\right)$ for the Legendre polynomials and performing integration with respect to the variables $-1 \leqslant t^{\prime} \leqslant 1,0 \leqslant \xi^{\prime} \leqslant \xi_{0}\left(t^{\prime}\right)$, where $\xi_{0}\left(t^{\prime}\right)$ is the root of equation $Y_{n}(\xi, \theta)=0$ at fixed $\theta$ and determines the equation of polytrope surface with a given index $n$, we obtain the relation

$$
\begin{aligned}
\Phi_{0}(\xi, \theta)-\Phi_{0}(0,0) & =\frac{\xi^{2}}{6}+\frac{\xi^{2}}{2} P_{2}(t) I_{2}+\sum_{l=2}^{\infty} P_{2 l}(t) \xi^{2 l} I_{2 l}, \\
I_{2} & =-\int_{-1}^{+1} P_{2}\left(t^{\prime}\right) \ln \left[\xi_{0}\left(t^{\prime}\right)\right] d t^{\prime}, \\
I_{2 l} & =(l-1)^{-1} \frac{1}{4} \int_{-1}^{+1} P_{2 l}\left(t^{\prime}\right)\left[\xi_{0}\left(t^{\prime}\right)\right]^{2-2 l} d t^{\prime} \quad \text { at } \quad l \geqslant 2 .
\end{aligned}
$$

Substituting expansion (23) into equation (22), we find that

$$
C_{2}=-\frac{\Omega^{2}}{6}\left(1+3 I_{2}\right), \quad C_{2 l}=-\Omega^{2} I_{2 l} \quad \text { at } \quad l \geqslant 2 .
$$

As a result, the integral equation (15) takes the form

$$
Y_{n}(\xi, \theta)=1+\frac{\Omega^{2} \xi^{2}}{6}\left(1-P_{2}(t)\right)+\frac{1}{4 \pi} \int Y_{n}^{n}\left(\xi^{\prime}, \theta^{\prime}\right) Q\left(\boldsymbol{\xi}, \boldsymbol{\xi}^{\prime}\right) d \boldsymbol{\xi}^{\prime}
$$

The system of equations (9) and (25) are closed, it does not require any additional information and determines the general solution $Y_{n}(\xi, \theta)$ that corresponds to conditions in the center and on configuration surface. We solve this system in the self-consistent way, which correctly describes the polytrope surface, unlike in works $[7,8,12]$.

\section{The Emden equation}

As was shown from the results of works [7-9,11,14-16], maximum values of the parameter $\left[\Omega_{\max }^{(n)}\right]^{2}$ are significantly less than one, therefore, the rotation plays role of correction at calculation the polytrope characteristics. The influence of rotation appears the most strongly on change of geometrical polytrope parameters, but these changes do not exceed $40 \%$. Because of this the zero approximation of equation (9) is the Emden equation [4]

$$
\Delta_{\xi} y_{n}(\xi)=-y_{n}^{n}(\xi)
$$

which describes the polytrope model without rotation with spherical symmetry of density distribution. Equation (26) corresponds to the boundary conditions $y_{n}(0)=1, d y_{n} / d \xi=0$ at $\xi=0$, as well as the condition $y_{n}(\xi) \geqslant 0$. By integrating with respect of the angular variables of the kernel $Q\left(\boldsymbol{\xi}, \boldsymbol{\xi}^{\prime}\right)$ of equation (25) at $\Omega=0$, we transform it to such form

$$
y_{n}(\xi)=1+\int_{0}^{\xi}\left\{\frac{\left(\xi^{\prime}\right)^{2}}{\xi}-\xi^{\prime}\right\} y_{n}^{n}\left(\xi^{\prime}\right) d \xi^{\prime}
$$

The properties of equation solutions (26), (27) are illustrated by known analytical expressions at $n=0,1$ and $5[22]$

$$
\begin{aligned}
& y_{0}(\xi)=1-\xi^{2} / 6, \quad \xi_{1}(0)=\sqrt{6} ; \\
& y_{1}(\xi)=\frac{\sin \xi}{\xi}, \quad \xi_{1}(1)=\pi ; \\
& y_{5}(\xi)=\left\{1+\xi^{2} / 3\right\}^{-1 / 2}, \quad \xi_{1}(5)=\infty .
\end{aligned}
$$

Mathematical Modeling and Computing, Vol. 8, No. 2, pp. 338-358 (2021) 
Here $\xi_{1}(n)$ is the dimensionless polytrope radius, the smaller root of equation $y(\xi)=0$ at fixed value of index $n$. The condition

$$
\int_{0}^{\xi_{1}(n)} y_{n}^{n}(\xi)\left(\xi-\frac{\xi^{2}}{\xi_{1}(n)}\right) d \xi=1
$$

is a kind of normalization condition. Because of that the subintegral function $y_{n}(\xi)$ is a decreasing positive function of $\xi$, from equality (29) it follows that $\xi_{1}(n)$ is a monotonically increasing function of index $n$. Dependence of functions $y_{n}(\xi)$ on index $n$ were calculated numerically and are illustrated in Fig. 1. These are alternating or oscillating functions, which have physical meaning in the range $0 \leqslant \xi \leqslant \xi_{1}(n)$, moreover the polytrope index can change continuously in the range $0 \leqslant n \leqslant 5$. In the region of small values of variable $\xi$ the Emden functions are represented by expansions

$$
y_{n}(\xi)=1-\frac{1}{3 !} \xi^{2}+\frac{n}{5 !} \xi^{4}-\frac{n(8 n-5)}{3 \cdot 7 !} \xi^{6}+\ldots,
$$

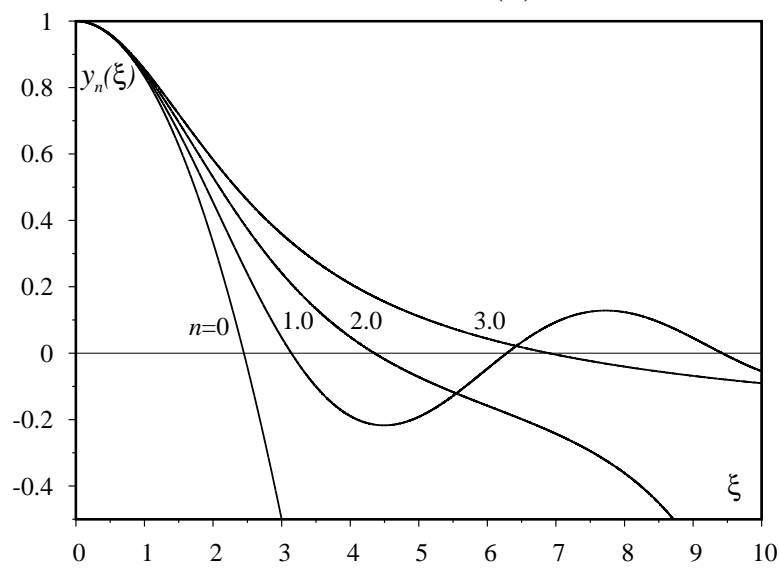

Fig. 1. The Emden solutions of equation for the polytropic index $0 \leqslant n \leqslant 3.0$.

from which we can see that with accuracy $\xi^{2}$ the asymptotics of functions $y_{n}(\xi)$ are the same, and it confirms the condition (12). Let us adduce here main characteristics of the polytropic stars in the Emden model. The mass

$$
\begin{aligned}
M(n, 0) & =\int_{V} \rho(\mathbf{r}) d \mathbf{r}=4 \pi \lambda_{n}^{3} \rho_{c} \beta_{2}(n), \\
\beta_{2}(n) & =\int_{0}^{\xi_{1}(n)} \xi^{2} y_{n}^{n}(\xi) d \xi=\xi_{1}^{2}(n)\left|\frac{d y_{n}}{d \xi}\right|_{\xi=\xi_{1}(n)},
\end{aligned}
$$

the volume of a star and its radius

$$
V(n, 0)=\frac{4 \pi}{3}\left(\lambda_{n} \xi_{1}(n)\right)^{3}, \quad R(n, 0)=\lambda_{n} \xi_{1}(n) ;
$$

moment of inertia relative to the axis of rotation

$$
I(n, 0)=\frac{8 \pi}{3} \rho_{c} \lambda_{n}^{5} \beta_{4}(n), \quad \beta_{4}(n)=\int_{0}^{\xi_{1}(n)} \xi^{4} y_{n}^{n}(\xi) d \xi
$$

The gravitational energy

$$
W(n, 0)=-\frac{G}{2} \int \rho\left(\mathbf{r}_{1}\right) d \mathbf{r}_{1} \int \rho\left(\mathbf{r}_{2}\right)\left|\mathbf{r}_{1}-\mathbf{r}_{2}\right|^{-1} d \mathbf{r}_{2}
$$

after integrating with respect to the angular variables and transition to the dimensionless variables and using equation (26) it is reduced to such form

$$
\begin{aligned}
W(n, 0) & =-48 \lambda_{n}^{5} \pi^{2} G \rho_{c}^{2} \frac{1}{1+n} J_{n} \\
J_{n} & =\int_{0}^{\xi_{1}(n)} \xi^{2} y_{n}^{n+1}(\xi) d \xi=\frac{n+1}{(5-n) \xi_{1}(n)} \beta_{2}^{2}(n), \quad n<5.0 .
\end{aligned}
$$

The total energy (gravitational and inner) equals [22]

$$
E(n, 0)=W(n, 0)+U(n, 0)=16 \pi^{2} G \lambda_{n}^{5} \frac{n-3}{(5-n) \xi_{1}(n)} \beta_{2}^{2}(n) .
$$

It follows that in single-phase model the polytropic star is stable at $n \leqslant 3$. 
Table 1. The parameters of the Emden polytropic models.

\begin{tabular}{|c|c|c|c|c|c|c|c|c|}
\hline$n$ & 0 & 0.25 & 0.5 & 1.0 & 1.5 & 2.0 & 2.5 & 3.0 \\
\hline \hline$\xi_{1}(n)$ & 2.4495 & 2.5921 & 2.7527 & 3.1416 & 3.6538 & 4.3529 & 5.3553 & 6.8969 \\
\hline$\beta_{2}(n)$ & 4.8990 & 4.2579 & 3.7887 & 3.1416 & 2.7141 & 2.4111 & 2.1872 & 2.0182 \\
\hline$\beta_{4}(n)$ & 17.6371 & 15.5178 & 14.0352 & 12.1567 & 11.1197 & 10.6110 & 10.5197 & 10.8516 \\
\hline
\end{tabular}

Dependence of the parameters $\xi_{1}(n), \beta_{2}(n), \beta_{4}(n)$ on the polytropic index $(0 \leqslant n \leqslant 3)$ is shown in Table 1 According to the formulae (31), (33) mass and moment of inertia of polytrope with index $n$ at the presence of rotation can be represented in the form

$$
M(n \mid \Omega)=M(n \mid 0) \eta(n \mid \Omega), \quad I(n \mid \Omega)=I(n \mid 0) \zeta(n \mid \Omega),
$$

where

$$
\begin{aligned}
& \eta(n \mid \Omega)=\left(2 \beta_{2}(n)\right)^{-1} \int_{-1}^{1} d t \int_{0}^{\xi_{0}(t)} \xi^{2} Y_{n}^{n}(\xi, \theta) d \xi \\
& \zeta(n \mid \Omega)=\left(\frac{4}{3} \beta_{4}(n)\right)^{-1} \int_{-1}^{1} d t\left(1-t^{2}\right) \int_{0}^{\xi_{0}(t)} \xi^{4} Y_{n}^{n}(\xi, \theta) d \xi
\end{aligned}
$$

Here $\xi_{0}(t)$ determines the polytrope surface of index $n$.

\section{The rotational polytrope $n=0$}

Equations (9) and (25) in general case are two-dimensional, twoparametric and nonlinear, since the index $n$ can take arbitrary interval values $(0 \div 5)$. In the case $n=0, n=1$ equations are linear, which allows to find approximate solutions in analytical form. It makes them important also in a methodological sense.

The polytrope $n=0$ describes a model with constant density. Therefore, in this case the problem reduced to determine the surface shape of rotational polytrope. According to equation (25) and relations (18) and (23) the condition $Y_{0}(\xi, \theta)=0$ is integral equation

$$
1-\frac{1}{6} \xi^{2}+\frac{\Omega^{2}}{6} \xi^{2}-\frac{P_{2}(t)}{6} \xi^{2}\left(\Omega^{2}+3 I_{2}\right)-\sum_{l \geqslant 2} \xi^{2 l} P_{2 l}(t) I_{2 l}=0 .
$$

It is easy to see, in the approximation $I_{2 l}=0$ at $l \geqslant 2$ the root of equation $(39) \xi_{0}(t)$ can be represent in the form of function, which describes the surface of rotational ellipsoid. For the purpose of self-consistent determination of ellipsoid parameters we choose a trial function in the form

$$
\xi_{0}^{(0)}(t \mid \Omega)=\xi_{e}^{(0)}\left\{1+\frac{e_{0}^{2}}{1-e_{0}^{2}} t^{2}\right\}^{-1 / 2} .
$$

From equation (39) in the mentioned approximation

$$
\xi_{0}^{(0)}(t \mid \Omega)=6^{1 / 2}\left\{1-\Omega^{2}+P_{2}(t)\left[\Omega^{2}+3 I_{2}^{(0)}\right]\right\}^{-1 / 2} .
$$

Equating the right sides of equations (40) and (41), we find equatorial radius

$$
\xi_{e}^{(0)}=6^{1 / 2}\left\{1-\frac{3}{2}\left(\Omega^{2}+I_{2}^{(0)}\right)\right\}^{-1 / 2}
$$

and the relation between eccentricity and angular velocity

$$
e_{0}^{2}+\left[3 e_{0}^{2}-\frac{9}{2}\right] I_{2}^{(0)}=\frac{3}{2} \Omega^{2}
$$


Using expression (40) for self-consistent calculation $I_{2}^{(0)}$, we find

$$
I_{2}^{(0)}=I_{2}^{(0)}\left(e_{0}\right)=\frac{2}{3}+\frac{1-e_{0}^{2}}{e_{0}^{2}}-\frac{\sqrt{1-e_{0}^{2}}}{e_{0}^{3}} \arcsin e_{0} .
$$

Expression (43) is known as the Maclaurin formula [23]. Dependencies of functions $e_{0}(\Omega)$ and $I_{2}^{(0)}(\Omega) \equiv$ $I_{2}^{(0)}\left(e_{0}(\Omega)\right)$ on the angular velocity are shown in Figs. 2 and 3.

According to the expression (44) at small values of eccentricity $I_{2}^{(0)}(e)=2 e^{2} / 15+$ $8 e^{4} / 105+\ldots$ In this approximation from the Maclaurin formula it follows, that at small angular velocities $e^{2}=15 \Omega^{2} / 4+\ldots$, and because of that

$$
I_{2}^{(0)}(\Omega)=\frac{1}{2} \Omega^{2}+\frac{45}{56} \Omega^{4}+\ldots
$$

It can be seen in Fig. 2, eccentricity is an ambiguous function of angular velocity. The maximal value of angular velocity $\Omega_{\max }=$ $0.47399 \ldots$, at which instability occurs, corresponds to eccentricity $e\left(\Omega_{\max }\right)=0.92995 \ldots$ In the region of large values of eccentricity

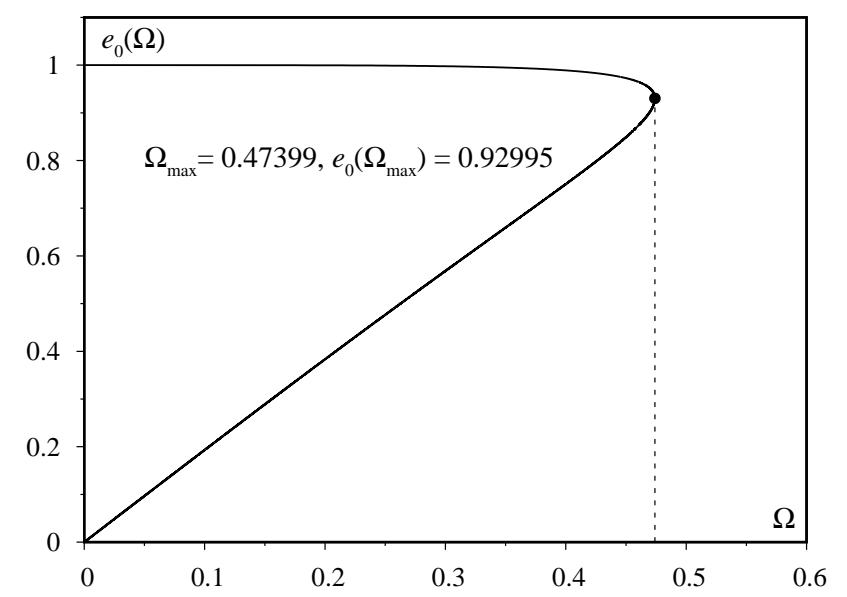

Fig. 2. Dependence of eccentricity $e_{0}(\Omega)$ on angular velocity $\Omega$. $\left(e\left(\Omega_{\max }\right) \leqslant e \leqslant 1\right)$ and small angular velocities

$$
I_{2}^{(0)}(\Omega) \approx \frac{2}{3}-\Omega^{2}-\Omega^{4}+\ldots
$$

which leads to the asymptotics

$$
\xi_{0}^{(0)}(t \mid \Omega) \cong \sqrt{2}\left\{\frac{\Omega^{4}}{2}+t^{2}\right\}^{-1 / 2}, \quad \xi_{e}(\Omega) \rightarrow \frac{2}{\Omega^{2}}+\ldots, \quad \xi_{p}(\Omega) \rightarrow \sqrt{2}
$$

which corresponds to the disk of constant thickness and large radius. Dependence $\xi_{e}^{(0)}(\Omega)$ and $\xi_{p}^{(0)}(\Omega)$ on angular velocity is shown in Fig. 4.

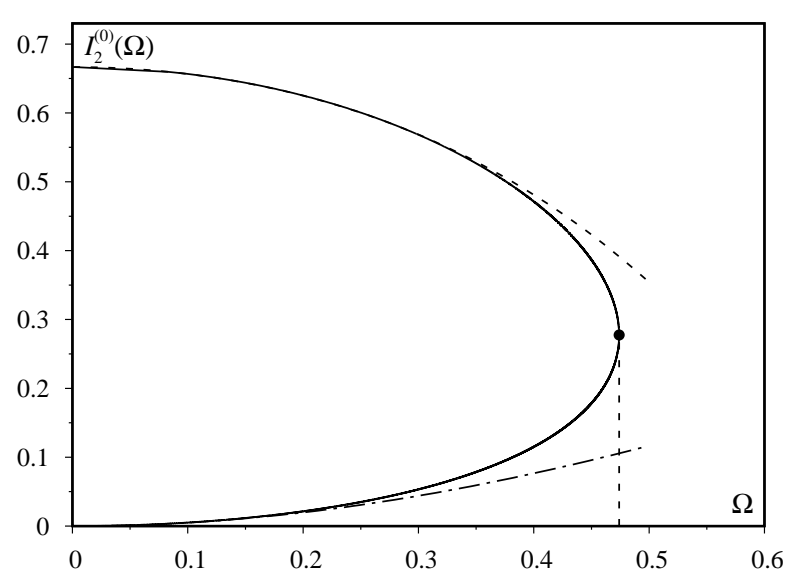

Fig. 3. Dependence of function $I_{2}^{(0)}(\Omega)$ on angular velocity $\Omega$. Dashed-dot curve corresponds to small velocity at small value of eccentricity, dashed curve corresponds to small velocity at large values of the eccentricity. $I_{2}^{(0)}\left(\Omega_{\max }\right)=0.27698$.

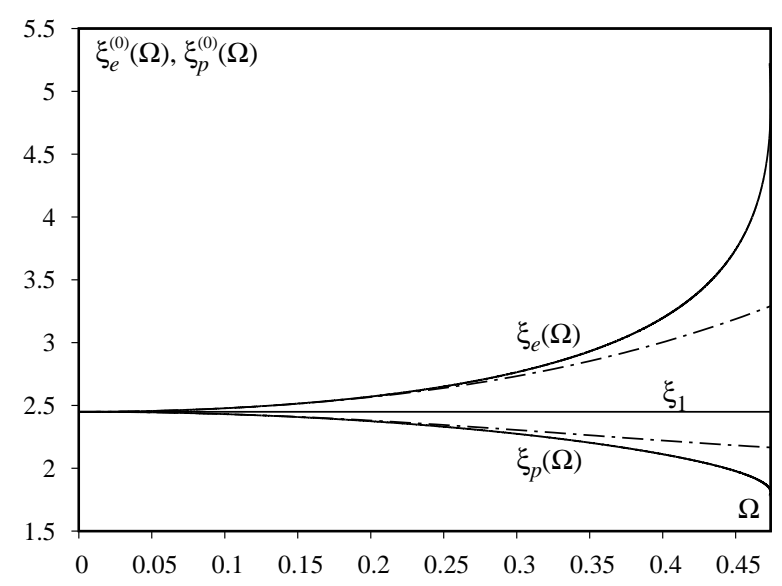

Fig. 4. Dependence of equatorial $\xi_{e}^{(0)}(\Omega)$ and polar $\xi_{p}^{(0)}(\Omega)$ radii on angular velocity $\Omega$ (the dashed-dot curve corresponds to small velocity). 


\section{What is the polytrope surface for $n=0$ ?}

As can be seen from above, in the approximation $I_{2 l}=0$ at $l \geqslant 2$ the surface of polytrope $n=0$ is the surface of rotational ellipsoid and is determined by expression (40). In Section 5 of this work it is shown, that the polytrope surface $n=1$ deviates from the surface of rotational ellipsoid and the value of this deviation increases with increasing calculation accuracy. Due to the fact that in equation

$$
1+\frac{\Omega^{2} \xi^{2}}{6}\left(1-P_{2}(t)\right)+\frac{1}{4 \pi} \int Q\left(\boldsymbol{\xi}, \boldsymbol{\xi}^{\prime}\right) d \boldsymbol{\xi}^{\prime}=0
$$

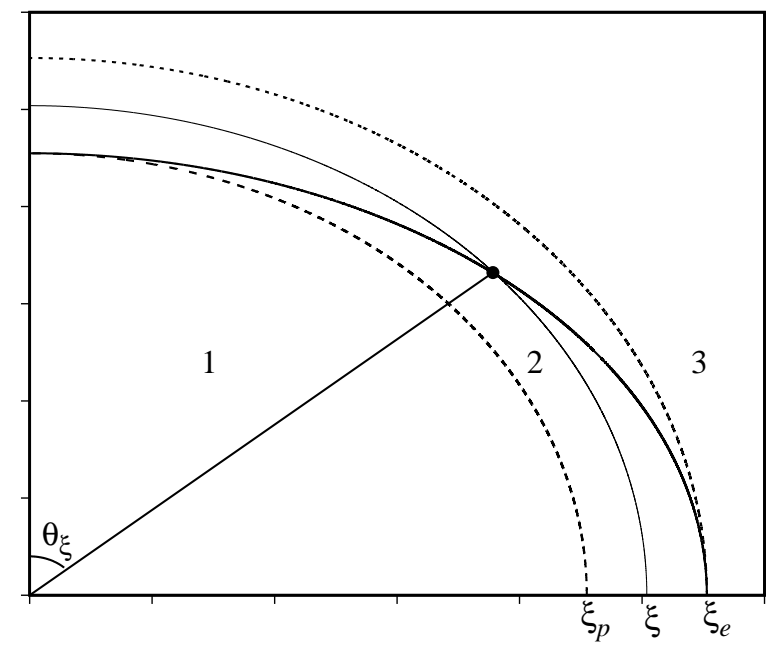

Fig. 5. Space regions in which gravitational potential has different representation. Solid curve shows the meridional section of the polytrope surface, which is in the region 2 .

which determines the polytrope surface $n=0$, integration over vector $\boldsymbol{\xi}^{\prime}$ is performed within this surface, then the equation (48) is nonlinear integral equation. as was shown in Fig. 5. And this equation can have solution, which is different from expression (40). To clarify this question, we applied iterative method of finding of equation solution (48), in which zero approximation for the surface $\xi_{0}(t)$ is different from (40). For this purpose we used expansions of gravitational potential in equation (48) for the Legendre polynomials in regions $1\left(0 \leqslant \xi \leqslant \xi_{p}\right), 2\left(\xi_{p} \leqslant \xi \leqslant \xi_{e}\right)$ and $3\left(\xi_{e}<\xi\right)$, where $\xi_{p}$ is the minimal distance from the origin to the surface, $\xi_{e}$ is the maximal, Solid curve represents the polytrope surface $\xi=\xi_{0}\left(t^{\prime}\right)$. In the region 1 the potential $\Phi_{0}(\xi, \theta)-\Phi_{0}(0,0)$ determined by formulas (23). In the region 3 we have standard multipole expansion of potential of external gravitational body,

$$
\frac{1}{4 \pi} \int Q\left(\boldsymbol{\xi}, \boldsymbol{\xi}^{\prime}\right) d \boldsymbol{\xi}^{\prime}=-\frac{1}{2} \int_{0}^{1} \xi_{0}^{2}\left(t^{\prime}\right) d t^{\prime}+\sum_{l \geqslant 0} \frac{P_{2 l}(t)}{(2 l+3) \xi^{2 l+1}} \int_{0}^{1} P_{2 l}\left(t^{\prime}\right)\left[\xi_{0}\left(t^{\prime}\right)\right]^{2 l+3} d t^{\prime} .
$$

In the region 2 the potential is superposition of expressions (23) and (48) and written in the form

$$
\begin{aligned}
\Phi_{0}(\xi, \theta)-\Phi_{0}(0,0)= & -\frac{1}{2} \int_{t}^{1} \xi_{0}^{2}\left(t^{\prime}\right) d t^{\prime}+\sum_{l \geqslant 0} \frac{P_{2 l}(t)}{(2 l+3) \xi^{2 l+1}} \int_{t}^{1} P_{2 l}\left(t^{\prime}\right)\left(\xi_{0}\left(t^{\prime}\right)\right)^{2 l+3} d t^{\prime} \\
& +\int_{0}^{t}\left\{-\frac{\xi^{2}}{6}+\xi^{2} P_{2}(t) P_{2}\left(t^{\prime}\right) \ln \left(\frac{\xi_{0}\left(t^{\prime}\right)}{\xi}\right)-\sum_{l \geqslant 2} \frac{P_{2 l}(t) \xi^{2 l}}{2(l-1)} \frac{P_{2 l}\left(t^{\prime}\right)}{\left[\xi_{0}\left(t^{\prime}\right)\right]^{2 l-2}}\right\} d t^{\prime} \\
& +\xi^{2} \int_{0}^{t}\left\{\sum_{l \geqslant 1} \frac{P_{2 l}(t) P_{2 l}\left(t^{\prime}\right)}{2 l+3}+\sum_{l \geqslant 2} \frac{P_{2 l}(t) P_{2 l}\left(t^{\prime}\right)}{2(l-1)}\right\} d t^{\prime} .
\end{aligned}
$$

At the pole point (at $t=1$ ) expression (50) continuously turns into expression (23), and in the vicinity of equator $(t=0)$ it turns into (49). Expression (50) determines the potential on sphere of radius $\xi$ both inside the polytrope and outside it in the region 2. Each sphere of radius $\xi$ crosses the polytrope surface in the circle of radius $\left(1-t^{2}\right)^{1 / 2} \xi_{0}(t)$. Putting in expression (50) $\xi=\xi_{0}(t)$, we obtain the value of gravitational potential on the sphere surface at a point with coordinates $\theta, \xi_{0}(\theta)$, or at points on the polytrope surface with coordinates $t, \xi_{0}(t)$.

Substituting expression (50) in equation (48) we obtained equation, the root of which determines the meridional section of the polytrope surface $\xi_{0}(t)$ at fixed $\Omega$. We solve this equation by the iterative method. In zero approximation only taken into account those terms of equation (48), in which appear $P_{2}(t)$ and $P_{2}\left(t^{\prime}\right)$. Found in this way the root $\tilde{\xi}_{0}(t)$ used for the next accounting terms, in which appear 
the Legendre polynomials of higher order. In Fig. 6 is shown the solution $\tilde{\xi}_{0}(t)$ at $\Omega=0.45$ (curve 1), as well as solutions, which correspond to accounting in the first order of perturbation theory of the terms with $P_{4}(t)$ (curve 2), $P_{6}(t)$ (curve 3 ) and $P_{8}(t)$ (curve 4 ). As was shown in Figure, there is a very good convergence on influence of the multipole terms of equation (48). To estimate the convergence, we use second term of equality (50)

$$
\Xi(t)=\sum_{l \geqslant 2} \frac{P_{2 l}(t)}{(2 l+3) \xi^{2 l+1}} \int_{t}^{1} P_{2 l}\left(t^{\prime}\right)\left(\xi_{0}\left(t^{\prime}\right)\right)^{2 l+3} d t^{\prime} .
$$

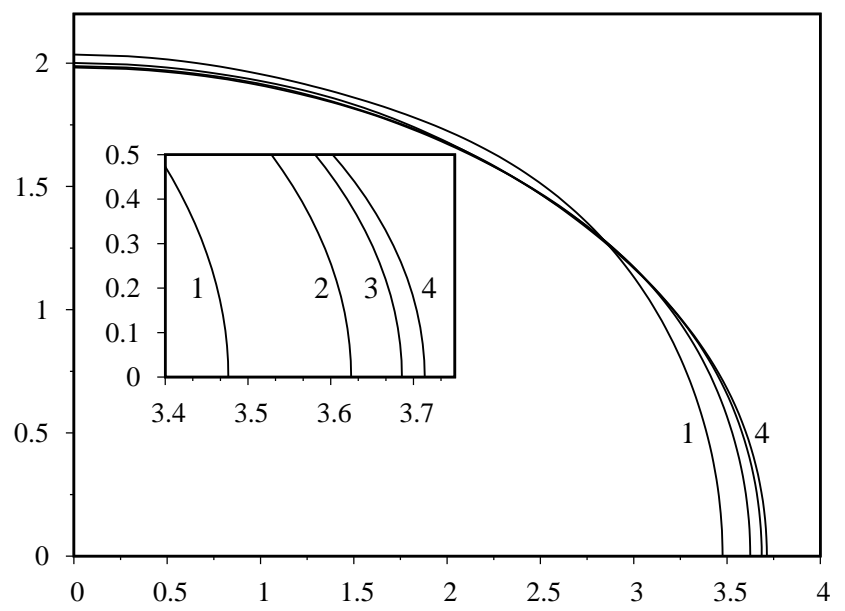

Fig. 6. The meridional section of the polytrope surface at $\Omega=0.45$. Curve 1 corresponds to accounting the terms with $P_{2}(t)$, curve 2: $P_{2}(t)$ and $P_{4}(t)$, curve 3 : $P_{2}(t)-P_{6}(t)$, curve 4: $P_{2}(t)-P_{8}(t)$.

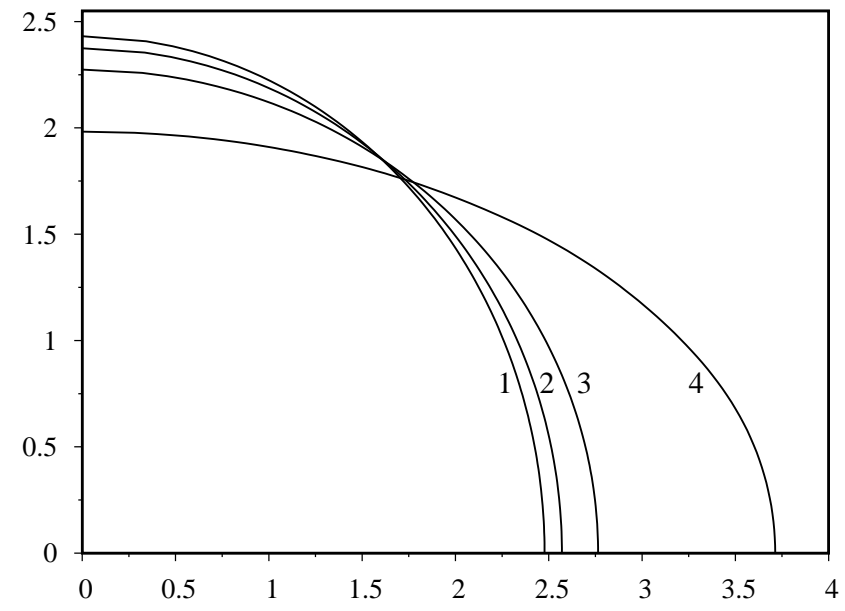

Fig. 7. The meridional section of the polytrope surface at different values of angular velocity $\Omega$. Curve 1 corresponds to $\Omega=0.1$, curve 2 : $\Omega=0.2$, curve 3 : $\Omega=0.3$, curve $4: \Omega=0.45$.

In the vicinity of equator, where $t$ is a small value

$$
\begin{aligned}
\Xi(t) & \approx \sum_{l \geqslant 2} \frac{P_{2 l}(t)}{(2 l+3) \xi^{2 l+1}} \int_{0}^{1} P_{2 l}\left(t^{\prime}\right)\left[\xi_{e}^{(0)}\right]^{2 l+3}\left\{1+\frac{e^{2}}{1-e^{2}}\left(t^{\prime}\right)^{2}\right\}^{-l-3 / 2} d t^{\prime}= \\
& =\sum_{l \geqslant 2}(-1)^{l} \frac{P_{2 l}(t)\left[\xi_{e}^{(0)}\right]^{2 l+3} e^{2 l}\left(1-e^{2}\right)^{1 / 2}}{\xi^{2 l+1}(2 l+1)(2 l+3)} \\
& \approx\left[\xi_{e}^{(0)}\right]^{2} \sum_{l \geqslant 2} \frac{\left|P_{2 l}(0)\right| e^{2 l}\left(1-e^{2}\right)^{1 / 2}}{(2 l+1)(2 l+3)}
\end{aligned}
$$

is expansion in powers of eccentricity, and all terms of the sum are positive. The solution, which corresponds to curve 4 is used for finding of solution in next iterations. The results of such calculation for several values of angular velocity $0.1 \leqslant \Omega \leqslant 0.45$ are shown in Fig. 7 .

In Table 2 it is shown the value of polar and equatorial radii of polytrope $n=0$, calculated by us, as well as found in work [13], for the same values $\Omega$, as in Fig. 7. As it turned out, the polytrope surface at any values $\Omega$, which calculated by iterative method, is approaching the surface of rotational ellipsoid, which is defined by formula (40), with increasing number of multipole terms in expansion of gravitational

Table 2. Dependence of polar and equatorial radii with index $n=0$ on angular velocity $\Omega$.

\begin{tabular}{|c|c|c|c|c|}
\hline$\Omega$ & $\xi_{p}(\Omega)$ & $\xi_{e}(\Omega)$ & $\xi_{p}(\Omega)[13]$ & $\xi_{e}(\Omega)[13]$ \\
\hline \hline 0.1 & 2.4310 & 2.4777 & 2.4220 & 2.4959 \\
\hline 0.2 & 2.3744 & 2.5708 & 2.3712 & 2.5746 \\
\hline 0.3 & 2.2744 & 2.7645 & 2.2733 & 2.7709 \\
\hline 0.4 & 2.1130 & 3.1926 & 2.1115 & 3.2021 \\
\hline 0.45 & 1.9826 & 3.7136 & 1.9745 & 3.7587 \\
\hline
\end{tabular}




\section{Polytrope $n=1$}

This model occupies an important place in general theory, because it manages to work out approximate methods of finding solutions of equation (9) for any $n$. With the help of substitution

$$
Y_{1}(\xi, \theta)=y_{1}(\xi)+\Omega^{2}\left\{\varphi(\xi, \theta)+\frac{\xi^{2}}{4} \sin ^{2} \theta\right\},
$$

where $y_{1}(\xi)=\sin \xi / \xi$ is the Emden function for $n=1$, equation (9) is reduced to equation for function $\varphi(\xi, \theta)$, which do not depend on parameter $\Omega$ :

$$
\Delta(\xi, \theta) \varphi(\xi, \theta)+\varphi(\xi, \theta)=-\frac{1}{4} \xi^{2} \sin ^{2} \theta .
$$

Solution of corresponding homogeneous equation in which variables are separated, can be represented in the form

where

$$
\varphi(\xi, \theta)=\sum_{l=1}^{\infty} \alpha_{2 l} j_{2 l}(\xi) P_{2 l}(t)
$$

$$
j_{2 l}(\xi)=\xi^{2 l} \sum_{s=0}^{\infty} \frac{1}{s !}\left(-\frac{\xi^{2}}{2}\right)^{s}\{[4 l+1+2 s] ! !\}^{-1}
$$

are the spherical Bessel functions of first kind [24], $P_{2 l}(t)$ are the Legendre polynomials on $t=\cos \theta$, $\alpha_{2 l}$ are integration constants.

Particular solution of equation (54) we represent in the form

$$
\varphi_{\text {part }}(\xi, \theta)=\sum_{l=2}^{\infty} b_{2 l}[\xi \sin \theta]^{2 l}
$$

Using the equality

$$
\Delta(\xi, \theta)\{\xi \sin \theta\}^{2 l}=(2 l)^{2}\{\xi \sin \theta\}^{2 l-2},
$$

we find the coefficients

$$
b_{2 l}=(-1)^{l-1} 2^{-2 l}(l !)^{-2}
$$

Therefore,

$$
\frac{1}{4} \xi^{2} \sin ^{2} \theta+\varphi_{\text {part }}(\xi, \theta)=1-J_{0}(\xi \sin \theta)
$$

where

$$
J_{0}(z)=\sum_{i=0}^{\infty}(-1)^{i}\left(\frac{z^{2}}{4}\right)^{i}(i !)^{-2}
$$

is the Bessel function of an integer (zero) order [24]. Thus the solution of equation (9) at $n=1$ takes the form

$$
Y_{1}(\xi, \theta)=j_{0}(\xi)+\Omega^{2}\left\{1-J_{0}(\xi \sin \theta)+\sum_{l=1}^{\infty} \alpha_{2 l} j_{2 l}(\xi) P_{2 l}(t)\right\} .
$$

The function $J_{0}(\xi \sin \theta)$ has such expansion for the Legendre polynomials [24]

$$
J_{0}\left(\xi\left[1-t^{2}\right]^{1 / 2}\right)=\sum_{l=0}^{\infty} D_{2 l} j_{2 l}(\xi) P_{2 l}(t), \quad D_{2 l}=(4 l+1)(2 l) ! 2^{-2 l}(l !)^{-2} .
$$

Because of solution (62) can be written also in the form of an ordinary expansion for the orthogonal functions

$$
\tilde{Y}_{1}(\xi, \theta)=j_{0}(\xi)+\Omega^{2}\left\{1-j_{0}(\xi)+\sum_{l=1}^{\infty} a_{2 l} j_{2 l}(\xi) P_{2 l}(t)\right\},
$$

where $a_{2 l}$ are new integration constants $\left(a_{2 l}=\alpha_{2 l}-D_{2 l}\right)$. Representations (62) and (64) are quite equivalent. In fact, at practical calculation it is necessary to restrict accounting the finite number of 
terms $\left(1 \leqslant l \leqslant l_{0}\right)$, therefore, the calculated polytrope characteristics are somewhat different from each other. Representation (62) can be considered as a result of selected summation of series (64). Note that the $J_{0}(z)$ has a very accurate analytical approximations [24], and

$$
\frac{1}{2} \int_{-1}^{+1} J_{0}\left(z\left[1-t^{2}\right]^{1 / 2}\right) d t=j_{0}(z) .
$$

Using expression (12) and equating the asymptotics of functions $Y_{0}(\xi, \theta)$ and $\tilde{Y}_{1}(\xi, \theta)$ at $\xi \ll 1$ (with accuracy to $\xi^{2}$ ), we find, that

$$
a_{2}(\Omega)=-\frac{5}{2}\left\{1+\frac{3}{\Omega^{2}} I_{2}(\Omega)\right\},
$$

where $I_{2}(\Omega)$ corresponds to the polytrope with index $n=0$ is an ambiguous function of $\Omega$. Therefore, in the region of small angular velocities and small eccentricities according to expression (45)

$$
a_{2}(\Omega)=-\frac{25}{4}\left\{1+\frac{27}{28} \Omega^{2}+\cdots\right\} .
$$

In the region of small angular velocities and eccentricities, which are close to one,

$$
\Omega^{2} a_{2}(\Omega) \rightarrow-5\left(1-\Omega^{2}+\ldots\right) .
$$

If we restricted the approximation $a_{2 l}=0$ at $l \geqslant 2$ in expansion (64), then in mode (67) the polytrope surface is the surface of rotational ellipsoid, which is close to the Emden sphere. Instead, in mode (68) the polytrope surface is the surface of strongly compressed rotational ellipsoid, indicating the formation of disk structure. Boundary forms of the meridional section of the polytrope surface with index $n=1$ at small angular velocities, which correspond to formulae (67) and (68), are shown in Fig. 8.

Since $Y_{1}(\xi, \theta)$ in form $(62)$ or $(64)$ is the solution of equation (26) at $n=1$, then this provides an alternative opportunity to determine integration constants. Let us show it on the example of expansion (64). Substituting it in equation (25), we reduce the last one to the following form

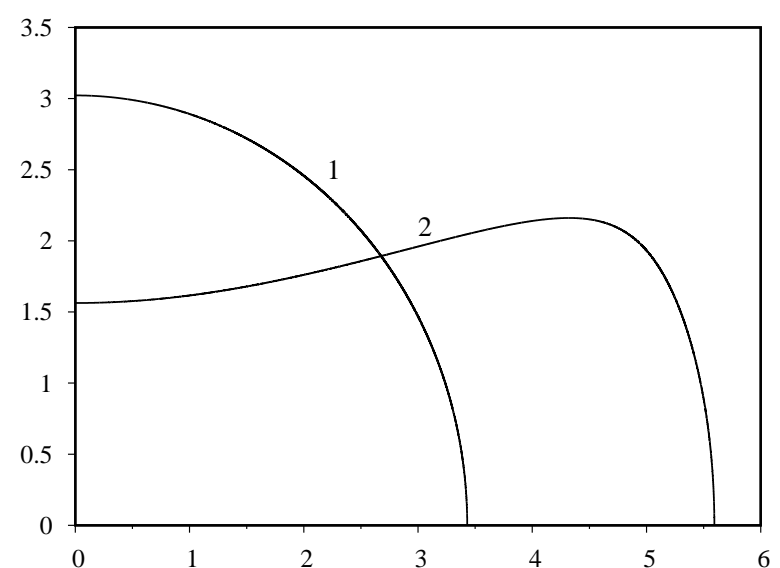

Fig. 8. The meridional section of the polytrope surface with index $n=1$ at fixed value of angular velocity $\Omega=0.2$. Curve 1 corresponds to approximation (67), curve 2: approximation (68).

$$
\begin{aligned}
\left(1-\Omega^{2}\right) j_{0}(\xi) & +\Omega^{2} \sum_{l \geqslant 1} a_{2 l} j_{2 l}(\xi) P_{2 l}(t)=1-\Omega^{2}-\Omega^{2}\left\{\Phi_{0}(\xi, \theta)-\Phi_{0}(0,0)\right\} \\
& +\frac{1}{4 \pi}\left(1-\Omega^{2}\right) \int Q\left(\boldsymbol{\xi}, \boldsymbol{\xi}^{\prime}\right) j_{0}\left(\boldsymbol{\xi}^{\prime}\right) d \boldsymbol{\xi}^{\prime}+\frac{\Omega^{2} \xi^{2}}{6}\left(1-P_{2}(t)\right) \\
& +\frac{1}{4 \pi} \Omega^{2} \sum_{l \geqslant 1} a_{2 l} \int Q\left(\boldsymbol{\xi}, \boldsymbol{\xi}^{\prime}\right) j_{2 l}\left(\boldsymbol{\xi}^{\prime}\right) P_{2 l}\left(t^{\prime}\right) d \boldsymbol{\xi}^{\prime}
\end{aligned}
$$

Integration over vector $\boldsymbol{\xi}^{\prime}$ is performed over the volume of polytrope. Therefore,

$$
\begin{aligned}
\frac{1}{4 \pi} \int Q\left(\boldsymbol{\xi}, \boldsymbol{\xi}^{\prime}\right) j_{0}\left(\boldsymbol{\xi}^{\prime}\right) d \boldsymbol{\xi}^{\prime}= & \int_{0}^{\xi} j_{0}\left(\boldsymbol{\xi}^{\prime}\right)\left\{\frac{\left(\xi^{\prime}\right)^{2}}{\xi}-\xi^{\prime}\right\} d \xi^{\prime} \\
& +\frac{1}{2} \sum_{l \geqslant 1} P_{2 l}(t) \xi^{2 l} \int_{-1}^{+1} P_{2 l}\left(t^{\prime}\right) \int_{\pi}^{\xi_{0}\left(t^{\prime}\right)} j_{0}\left(\xi^{\prime}\right)\left(\xi^{\prime}\right)^{1-2 l} d \xi^{\prime},
\end{aligned}
$$

where $\xi_{0}\left(t^{\prime}\right)$ determines the equation of the polytrope surface. Taking into account the following, the 
$j_{0}(\xi)$ satisfies equation (27), and taking into account expression (23), we rewrite equality (69) in such form

$$
\begin{aligned}
\sum_{l \geqslant 1} a_{2 l} j_{2 l}(\xi) P_{2 l}(t)= & -P_{2}(t) \frac{\xi^{2}}{6}\left(1+3 I_{2}\right)-\sum_{l \geqslant 2} P_{2 l}(t) \xi^{2 l} I_{2 l} \\
& +\sum_{l \geqslant 1} L_{2 l} \xi^{2 l} P_{2 l}(t)+\frac{1}{4 \pi} \sum_{l \geqslant 1} a_{2 l} \int Q\left(\boldsymbol{\xi}, \boldsymbol{\xi}^{\prime}\right) j_{2 l}\left(\boldsymbol{\xi}^{\prime}\right) P_{2 l}\left(t^{\prime}\right) d \boldsymbol{\xi}^{\prime} .
\end{aligned}
$$

Herewith $I_{2 l}$ are determined by formulae (23), and

$$
L_{2 l}=\frac{\Omega^{-2}}{2} \int_{-1}^{+1} P_{2 l}\left(t^{\prime}\right) \int_{\pi}^{\xi_{0}\left(t^{\prime}\right)} j_{0}\left(\xi^{\prime}\right)\left(\xi^{\prime}\right)^{1-2 l} d \xi^{\prime} d t^{\prime} .
$$

As noted above, in approximation (40) the coefficients $I_{2 l}=0$ at $l \geqslant 2$. However $L_{2 l}$ and in this approximation are nonzero, although in our previous publications [14-16] they were not taken into account, as well as $I_{2 l}$.

Calculation of the last term on the right side of equality (71) are carried out by expansion of kernel $Q\left(\boldsymbol{\xi}, \boldsymbol{\xi}^{\prime}\right)$ for the Legendre polynomials,

$$
\begin{aligned}
& \frac{1}{4 \pi} \int Q\left(\boldsymbol{\xi}, \boldsymbol{\xi}^{\prime}\right) j_{2 l}\left(\xi^{\prime}\right) P_{2 l}\left(t^{\prime}\right) d \boldsymbol{\xi}^{\prime}=P_{2 l}(t)(4 l+1)^{-1} \xi^{-1-2 l} \int_{0}^{\xi}\left(\xi^{\prime}\right)^{2+2 l} j_{2 l}\left(\xi^{\prime}\right) d \xi^{\prime} \\
& \quad+\frac{1}{2} P_{2 l}(t) \xi^{2 l} \int_{-1}^{+1} P_{2 l}^{2}\left(t^{\prime}\right) d t^{\prime} \int_{\xi}^{\xi_{0}\left(t^{\prime}\right)} j_{2 l}\left(\xi^{\prime}\right)\left(\xi^{\prime}\right)^{1-2 l} d \xi^{\prime} \\
& \quad+\frac{1}{2} \sum_{m=1}^{\infty} P_{2 m}(t) \xi^{2 m}\left(1-\delta_{m, l}\right) \int_{-1}^{+1} d t^{\prime} P_{2 l}\left(t^{\prime}\right) P_{2 m}\left(t^{\prime}\right) \int_{\pi}^{\xi_{0}\left(t^{\prime}\right)} j_{2 l}\left(\xi^{\prime}\right)\left(\xi^{\prime}\right)^{1-2 m} d \xi^{\prime},
\end{aligned}
$$

where $\delta_{m, l}$ is the Kronecker symbol, and $\xi_{0}\left(t^{\prime}\right)$ determines the surface equation. Integration with respect to the variable $\xi^{\prime}$ in the first two terms of right side of equality (73) is performed in analytical form,

$$
\begin{aligned}
& \xi^{-1-2 l} \int_{0}^{\xi}\left(\xi^{\prime}\right)^{2+2 l} j_{2 l}\left(\xi^{\prime}\right) d \xi^{\prime}=\xi j_{2 l+1}(\xi) \\
& \int_{\xi}^{\xi_{0}} j_{2 l}\left(\xi^{\prime}\right)\left(\xi^{\prime}\right)^{1-2 l} d \xi^{\prime}=-\xi_{0}^{1-2 l} j_{2 l-1}\left(\xi_{0}\right)+\xi^{1-2 l} j_{2 l-1}(\xi),
\end{aligned}
$$

where $\xi_{0} \equiv \xi_{0}\left(t^{\prime}\right)$. Taking into account relation

$$
j_{2 l}(\xi)=[4 l+1]^{-1} \xi\left\{j_{2 l+1}(\xi)+j_{2 l-1}(\xi)\right\},
$$

we see, that the sum of first two terms of right side of equality (73) can be rewritten in the form

$$
P_{2 l}(t) j_{2 l}(\xi)-P_{2 l}(t) \xi^{2 l}\left\{\int_{0}^{1} P_{2 l}^{2}\left(t^{\prime}\right) \xi_{0}^{1-2 l}\left(t^{\prime}\right) j_{2 l-1}\left(\xi_{0}\left(t^{\prime}\right)\right) d t^{\prime}\right\} .
$$

As a result, the terms of equation (71), in which $j_{2 l}(\xi)$ appear explicitly, are mutually compensated and remain only the terms of type $\xi^{2 l} P_{2 l}(t), \xi^{2 m} P_{2 m}(t)$.

Comparing the coefficients at the same products $\xi^{2 l} P_{2 l}(t)$ in equation, we obtain the system of linear inhomogeneous algebraic equations for integration constants $a_{2 l}$

$$
\begin{aligned}
& a_{2} S_{2,2}+a_{4} S_{2,4}+\ldots+a_{2 l_{0}} S_{2,2 l_{0}}=-\frac{1}{6}\left(1+3 I_{2}\right)+L_{2}, \\
& a_{2} S_{4,2}+a_{4} S_{4,4}+\ldots+a_{2 l_{0}} S_{4,2 l_{0}}=L_{4}-I_{4} ; \\
& a_{2} S_{2 l_{0}, 2}+a_{4} S_{2 l_{0}, 4}+\ldots+a_{2 l_{0}} S_{2 l_{0}, 2 l_{0}}=L_{2 l_{0}}-I_{2 l_{0}} .
\end{aligned}
$$


Matrix elements $S_{2 l, 2 l}, S_{2 m, 2 l}$ are determined by expressions

$$
\begin{aligned}
S_{2 l, 2 l} & =\int_{0}^{1} P_{2 l}^{2}(t) \xi_{0}^{1-2 l} j_{2 l-1}\left(\xi_{0}\right) d t \\
S_{2 m, 2 l} & =-\int_{0}^{1} P_{2 m}(t) P_{2 l}(t) d t \int_{\pi}^{\xi_{0}}\left(\xi^{\prime}\right)^{1-2 m} j_{2 l}\left(\xi^{\prime}\right) d \xi^{\prime} .
\end{aligned}
$$

After integration with respect to the variable $\xi^{\prime}$ non-diagonal elements are also reduced to single integrals

$$
\begin{aligned}
S_{2,4} & =\int_{0}^{1} P_{2}(t) P_{4}(t) \xi_{0}^{-1}\left\{j_{3}\left(\xi_{0}\right)+2 \xi_{0}^{-1} j_{2}\left(\xi_{0}\right)\right\} d t \\
S_{2,6} & =\int_{0}^{1} P_{2}(t) P_{6}(t) \xi_{0}^{-1}\left\{j_{5}\left(\xi_{0}\right)+4 \xi_{0}^{-1} j_{4}\left(\xi_{0}\right)+8 \xi_{0}^{-2} j_{3}\left(\xi_{0}\right)\right\} d t .
\end{aligned}
$$

At small values of angular velocity in zero approximation in expressions (78), (79) it is enough to replace $\xi_{0}(t)$ on dimensionless Emden radius with $\xi_{1}=\pi$, resulting

$$
\begin{aligned}
S_{2 l, 2 l} & \Rightarrow S_{2 l, 2 l}^{(0)}=(4 l+1)^{-1} \xi_{1}^{1-2 l} j_{2 l-1}\left(\xi_{1}\right) ; \\
S_{2 l, 2 m} & \Rightarrow S_{2 l, 2 m}^{(0)}=0 \quad \text { at } \quad l \neq m .
\end{aligned}
$$

In such approximation $I_{2 l}=L_{2 l}=0$ at $l \geqslant 1$,

$$
a_{2}^{(0)}=-\frac{5}{6} \pi^{2}, \quad a_{2 l}^{(0)}=0 \quad \text { at } \quad l \geqslant 2,
$$

which coincides with the result of work [8]. In this work the approximate solution of equilibrium equation is presented in the form

$$
Y_{1}^{\mathrm{Ch}}(\xi, \theta)=y_{1}(\xi)+\Omega^{2}\left\{\psi_{0}(\xi)+a_{2} P_{2}(t) \psi_{2}(\xi)\right\}
$$

and integration constant $a_{2}$ found by the Milne method [7] from condition of continuity of the gravitational potential on the Emden surface $\xi=\xi_{1}(1)=\pi$. At the same time functions $\psi_{0}(\xi)$ and $\psi_{2}(\xi)$ found by numerical integration of the corresponding linear differential equations in the region $0 \leqslant \xi \leqslant \xi_{1}(1)$. Equation of the polytrope surface found from condition $Y_{1}^{\mathrm{Ch}}(\xi, \theta)=0$, which at $y_{1}\left(\xi_{1}\right)=0$ takes the form

$$
\left(d y_{1} / d \xi\right)_{\xi=\xi_{1}}\left(\xi_{0}-\xi_{1}\right)+\Omega^{2}\left\{\psi_{0}\left(\xi_{1}\right)+a_{2} \psi_{2}\left(\xi_{1}\right) P_{2}(t)\right\} \simeq 0 .
$$

According to formula $(63) \psi_{0}\left(\xi_{1}\right)=1-j_{0}\left(\xi_{1}\right)=1, \psi_{2}\left(\xi_{1}\right)=j_{2}\left(\xi_{1}\right)=3 / \pi^{2}$, therefore [8]

$$
\xi_{0}(t) \approx \pi\left\{1+\Omega^{2}\left[1-\frac{5}{2} P_{2}(t)\right]\right\} .
$$

In the work [15] we solved the system of equations (77) by method of numerical iterations at $l_{0}=3$. Herewith integration over the polytrope volume performed within the rotational ellipsoid (see form. (40)) with some eccentricity and equatorial radius, which were found self-consistently. In zero iteration constants $a_{2 l}$ chosen in the form (81). In the frame of $i$-th iteration

$$
Y_{1}^{(i)}(\xi, \theta)=j_{0}(\xi)+\Omega^{2}\left\{1-j_{0}(\xi)+\sum_{l=1}^{3} a_{2 l}^{(i)} P_{2 l}(t) j_{2 l}(\xi)\right\},
$$

equatorial $\xi_{e}^{(i)}(\Omega)$ and polar $\xi_{p}^{(i)}(\Omega)$ radii are determined numerically from condition $Y_{1}^{(i)}(\xi, \theta)=0$, and eccentricity - from condition $e_{i}(\Omega)=\left\{1-\left[\xi_{p}^{(i)}(\Omega) / \xi_{e}^{(i)}(\Omega)\right]^{2}\right\}^{1 / 2}$. Coefficients of matrix $S_{2 l, 2 l}^{(i)}, S_{2 l, 2 m}^{(i)}$ are determined by formulae $(78)$ at $\xi_{0}^{(i)}(t)=\xi_{e}^{(i)}(\Omega)\left\{1+t^{2} e_{i}^{2}(\Omega)\left[1-e_{i}^{2}(\Omega)\right]^{-1}\right\}^{-1}$ and used for next iteration. Found in this way constants $a_{2 l}(\Omega)$ for some value $\Omega_{1}$ are used as zero approximation for $\Omega_{2}=\Omega_{1}+\Delta \Omega$. Herewith the coefficients $L_{2 l}$ in equations (77) were neglected, and $I_{2 l}=0$ at $l \geqslant 2$ due 
to the approximation of the rotational ellipsoid model. The results of calculations for dependencies of equatorial and polar radii, eccentricity, mass and moment of inertia of polytrope on angular velocity were shown in Table 3 . Note, that the used algorithm is applicable to the description of typically ellipsoidal configurations, for which are typical not very large eccentricities. In Table was shown, that the maximal value of angular velocity $\Omega_{\max }(1)$ approximately equals to $0.246 \ldots$, and eccentricity varies within $0 \leqslant e \leqslant 0.7868 \ldots$ Rotation leads to the relative increasing equatorial radius, that does not exceed $41 \%$, and the relative decreasing of polar radius does not exceed $14.3 \%$. Increasing of total mass due to rotation does not exceed $26 \%$, but moment of inertia relative to the axis of rotation increase almost in 2 times. As was shown in Table, taking into account terms $a_{2 l} P_{2 l}(t) j_{2 l}(\xi)$ at $l \geqslant 2$ is insignificant in the region $0<\Omega \leqslant 1 / 3 \Omega_{\max }(1)$, which determines the scope of the results of works $[7,8]$ and other. Also note, that expansion (64) is alternating, which testifies to its convergence, even in the region $2 / 3<\Omega / \Omega_{\max }<1$ coefficients $a_{2}(\Omega), a_{4}(\Omega), a_{6}(\Omega)$ are commensurate with each other for modulo.

Table 3. Dependence of the model characteristics with index $n=1$ on angular velocity according to representation (64) in approximation $a_{2 l}=0$ at $l \geqslant 4$ and $L_{2 l}=0$ for $l \geqslant 1$.

\begin{tabular}{|c|c|c|c|c|c|c|c|c|}
\hline$\Omega$ & $e(\Omega)$ & $\xi_{p}(\Omega)$ & $\xi_{e}(\Omega)$ & $a_{2}(\Omega)$ & $a_{4}(\Omega)$ & $a_{6}(\Omega)$ & $\eta(n, \Omega)$ & $\zeta(n, \Omega)$ \\
\hline 0.01000 & 0.02739 & 3.14112 & 3.14230 & -8.22784 & 0.00610775 & $-8.02713 \cdot 10^{-6}$ & 1.00023 & 1.00062 \\
\hline 0.02000 & 0.05478 & 3.13971 & 3.14443 & -8.23739 & 0.02449 & -0.000128907 & 1.00092 & 1.00249 \\
\hline 0.03000 & 0.08219 & 3.13734 & 3.14799 & -8.25338 & 0.055325 & -0.000656943 & 1.00207 & 1.00563 \\
\hline 0.04000 & 0.10961 & 3.13402 & 3.15302 & -8.27594 & 0.0989151 & -0.00209575 & 1.00369 & 1.01006 \\
\hline 0.05000 & 0.13706 & 3.12973 & 3.15955 & -8.30523 & 0.155695 & -0.0051788 & 1.00580 & 1.01583 \\
\hline 0.06000 & 0.16455 & 3.12447 & 3.16765 & -8.34151 & 0.226242 & -0.0108998 & 1.00839 & 1.02298 \\
\hline 0.07000 & 0.19208 & 3.11820 & 3.17737 & -8.38505 & 0.311294 & -0.020555 & 1.01150 & 1.03158 \\
\hline 0.08000 & 0.21967 & 3.11092 & 3.18880 & -8.43625 & 0.411773 & -0.0358001 & 1.01513 & 1.04172 \\
\hline 0.09000 & 0.24733 & 3.10259 & 3.20205 & -8.49557 & 0.52881 & -0.0587258 & 1.01933 & 1.05351 \\
\hline 0.10000 & 0.27507 & 3.09318 & 3.21725 & -8.56357 & 0.663789 & -0.0919578 & 1.02410 & 1.06707 \\
\hline 0.11000 & 0.30291 & 3.08266 & 3.23456 & -8.64098 & 0.818398 & -0.13879 & 1.02951 & 1.08256 \\
\hline 0.12000 & 0.33087 & 3.07097 & 3.25416 & -8.72865 & 0.9947 & -0.203359 & 1.03557 & 1.10016 \\
\hline 0.13000 & 0.35900 & 3.05807 & 3.27632 & -8.82768 & 1.19523 & -0.290887 & 1.04237 & 1.12011 \\
\hline 0.14000 & 0.38731 & 3.04388 & 3.30131 & -8.93941 & 1.42314 & -0.408009 & 1.04994 & 1.14270 \\
\hline 0.15000 & 0.41586 & 3.02832 & 3.32953 & -9.06557 & 1.68239 & $\begin{array}{c}-563239 \\
\end{array}$ & 1.05839 & 1.16830 \\
\hline$\overline{0.16000}$ & 0.44471 & 3.01127 & 3.36147 & -9.20840 & 1.97802 & -0.767633 & 1.06782 & 1.19736 \\
\hline 0.17000 & 0.47394 & 2.99259 & 3.39779 & -9.37084 & 2.31667 & -1.03579 & 1.07834 & 1.23047 \\
\hline 0.18000 & 0.50367 & 2.97208 & 3.43938 & -9.55694 & 2.70721 & -1.3874 & 1.09014 & 1.26843 \\
\hline 0.19000 & 0.53407 & 2.94946 & 3.48752 & -9.77240 & 3.16206 & -1.84985 & 1.10343 & 1.31232 \\
\hline 0.20000 & 0.56538 & 2.92430 & 3.54414 & -10.02570 & 3.69946 & -2.46274 & 1.11855 & 1.36371 \\
\hline$\overline{0.21000}$ & 0.59802 & 2.89594 & 3.61237 & -10.33050 & 4.3481 & -3.28708 & 1.13597 & 1.42496 \\
\hline 0.22000 & 0.63273 & 2.86321 & 3.69793 & -10.71110 & 5.15825 & -4.42644 & 1.15648 & 1.50007 \\
\hline 0.23000 & 0.67114 & 2.82368 & 3.81334 & -11.21930 & 6.23501 & -6.09077 & 1.18158 & 1.59696 \\
\hline 0.24000 & 0.71852 & 2.77019 & 4.00008 & -12.01930 & 7.90279 & -8.92229 & 1.21544 & 1.73805 \\
\hline$\overline{0.24100}$ & 0.72446 & 2.76320 & 4.02826 & -12.13670 & 8.14357 & -9.35381 & 1.21980 & 1.75727 \\
\hline$\overline{0.24200}$ & 0.73086 & 2.75562 & 4.06018 & -12.26860 & 8.41224 & -9.84267 & 1.22449 & 1.77830 \\
\hline 0.24300 & 0.73793 & 2.74724 & 4.09737 & -12.42040 & 8.71955 & -10.4124 & 1.22964 & 1.80179 \\
\hline$\overline{0.24400}$ & 0.74604 & 2.73767 & 4.14281 & -12.60320 & 9.0861 & -11.1087 & 1.23546 & 1.82894 \\
\hline 0.24500 & 0.75612 & 2.72593 & 4.20403 & -12.84440 & 9.5632 & -12.0491 & 1.24249 & 1.86270 \\
\hline 0.24600 & 0.77450 & 2.70593 & 4.33124 & -13.32470 & 10.4868 & -14.0363 & 1.25413 & 1.92196 \\
\hline 0.24601 & 0.77507 & 2.70536 & 4.33555 & -13.34070 & 10.5167 & -14.1062 & 1.25445 & 1.92369 \\
\hline 0.24602 & 0.77563 & 2.70481 & 4.33977 & -13.35610 & 10.5455 & -14.1737 & 1.25477 & 1.92537 \\
\hline 0.24603 & 0.77626 & 2.70418 & 4.34461 & -13.37360 & 10.5784 & -14.2512 & 1.25512 & 1.92728 \\
\hline 0.24604 & 0.77702 & 2.70344 & 4.35043 & -13.39470 & 10.6177 & -14.3446 & 1.25554 & 1.92955 \\
\hline 0.24605 & 0.77800 & 2.70249 & 4.35808 & -13.42230 & 10.669 & -14.4675 & 1.25608 & 1.93248 \\
\hline 0.24606 & 0.77959 & 2.70100 & 4.37053 & -13.46670 & 10.7512 & -14.6673 & 1.25693 & 1.93714 \\
\hline 0.24607 & 0.78685 & 2.69478 & 4.42985 & -13.66320 & 11.1084 & -15.5733 & 1.26065 & 1.95773 \\
\hline
\end{tabular}


Table 4. Dependence of the model characteristics with index $n=1$ on angular velocity according to representation (64) in approximation $a_{2 l}=0$ and $L_{2 l}=0$ at $l \geqslant 3$.

\begin{tabular}{|c|c|c|c|c|c|c|c|}
\hline$\Omega$ & $e(\Omega)$ & $\xi_{p}(\Omega)$ & $\xi_{e}(\Omega)$ & $a_{2}(\Omega)$ & $a_{4}(\Omega)$ & $\eta(n, \Omega)$ & $\zeta(n, \Omega)$ \\
\hline 0.01000 & $\overline{0.02740}$ & 3.14112 & 3.14230 & -8.22626 & 0.00305293 & 1.00023 & 1.00062 \\
\hline 0.02000 & 0.05477 & 3.13971 & 3.14443 & -8.23103 & 0.0122298 & 1.00092 & 1.00249 \\
\hline 0.03000 & 0.08211 & 3.13735 & 3.14798 & -8.23900 & 0.0275852 & 1.00207 & 1.00563 \\
\hline 0.04000 & 0.10947 & 3.13404 & 3.15299 & -8.25022 & 0.0492111 & 1.00369 & 1.01005 \\
\hline 0.05000 & 0.13679 & 3.12979 & 3.15949 & -8.26474 & 0.0772383 & 1.0058 & 1.01580 \\
\hline 0.06000 & 0.16406 & 3.12459 & 3.16751 & -8.28265 & 0.111839 & 1.00839 & 1.02292 \\
\hline 0.07000 & 0.19132 & 3.11842 & 3.17711 & -8.30402 & 0.153229 & 1.01149 & 1.03147 \\
\hline 0.08000 & 0.21851 & 3.11130 & 3.18835 & -8.32899 & 0.201673 & 1.01513 & 1.04152 \\
\hline 0.09000 & 0.24567 & 3.10320 & 3.20131 & -8.35768 & 0.257487 & 1.01931 & 1.05317 \\
\hline 0.10000 & 0.27276 & 3.09413 & 3.21608 & -8.39027 & 0.32105 & 1.02408 & 1.06652 \\
\hline 0.11000 & 0.29980 & 3.08407 & 3.23277 & -8.42695 & 0.392803 & 1.02945 & 1.08170 \\
\hline 0.12000 & 0.32678 & 3.07301 & 3.25152 & -8.46795 & 0.473268 & 1.03548 & 1.09886 \\
\hline 0.13000 & 0.35369 & 3.06094 & 3.27247 & -8.51353 & 0.563055 & 1.04221 & 1.11819 \\
\hline 0.14000 & 0.38056 & 3.04784 & 3.29583 & -8.56402 & 0.662877 & 1.04969 & 1.13989 \\
\hline 0.15000 & 0.40737 & 3.03369 & 3.32181 & -8.61978 & 0.773574 & 1.05798 & 1.16424 \\
\hline 0.16000 & 0.43413 & 3.01847 & 3.35069 & -8.68124 & 0.896136 & 1.06716 & 1.19153 \\
\hline 0.17000 & 0.46085 & 3.00216 & 3.38279 & -8.74892 & 1.03173 & 1.07731 & 1.22215 \\
\hline 0.18000 & 0.48754 & 2.98472 & 3.41852 & -8.82344 & 1.18177 & 1.08855 & 1.25656 \\
\hline 0.19000 & 0.51422 & 2.96611 & 3.45838 & -8.90554 & 1.34793 & 1.10099 & 1.29532 \\
\hline 0.20000 & 0.54093 & 2.94628 & 3.50303 & -8.99612 & 1.53227 & 1.1148 & 1.33915 \\
\hline 0.21000 & 0.56770 & 2.92519 & 3.55328 & -9.09629 & 1.73734 & 1.13016 & 1.38894 \\
\hline 0.22000 & 0.59459 & 2.90275 & 3.61026 & -9.20745 & 1.96635 & 1.14732 & 1.44585 \\
\hline 0.23000 & 0.62169 & 2.87887 & 3.67547 & -9.33137 & 2.22345 & 1.16657 & 1.51142 \\
\hline 0.24000 & 0.64911 & 2.85344 & 3.75110 & -9.47041 & 2.51415 & 1.18832 & 1.58773 \\
\hline 0.25000 & 0.67706 & 2.82630 & 3.84047 & -9.62772 & 2.84613 & 1.21311 & 1.67772 \\
\hline 0.26000 & 0.70588 & 2.79723 & 3.94905 & -9.80780 & 3.2307 & 1.24172 & 1.78576 \\
\hline 0.27000 & 0.73624 & 2.76593 & 4.08725 & -10.01740 & 3.68631 & 1.27531 & 1.91880 \\
\hline 0.28000 & 0.76981 & 2.73197 & 4.28026 & -10.26740 & 4.24909 & 1.31586 & 2.08937 \\
\hline 0.29000 & 0.81612 & 2.69556 & 4.66457 & -10.56940 & 5.04888 & 1.3676 & 2.32692 \\
\hline 0.29100 & 0.82627 & 2.69274 & 4.78049 & -10.59160 & 5.18552 & 1.37363 & 2.35676 \\
\hline 0.29110 & 0.82808 & 2.69267 & 4.80312 & -10.59110 & 5.20549 & 1.3742 & 2.35965 \\
\hline
\end{tabular}

In Table 4 was shown the results of calculation of the polytrope characteristics with $n=1$ as functions of angular velocity by taking into account coefficients $L_{2 l}$ (see form. (72)) in approximation $a_{2 l}=0$ at $l \geqslant 3$. In this approximation the maximal value of angular velocity is approximately $0.291 \ldots$, which is very close to the result of work [9], in which the numerical integration of equilibrium equation was performed. Taking into account of coefficients $L_{2 l}$ significantly changes the polytrope characteristics at $n=1$. Comparison of found integration constants $a_{2}(\Omega)$ and $a_{4}(\Omega)$ indicate better convergence of expansion (64) than in approximation, that do not take into account coefficients $L_{2 l}$. The constant $a_{2}(\Omega)$ for modulo and $a_{4}(\Omega)$ significantly decreased. In general, in both variants of calculation the dependence of integration constants on angular velocity is significant, as can be seen in Tables 3 and 4 . Taking into account of coefficients $L_{2 l}$ practically does not affect the value of polar radius, but increase equatorial radius, as well as mass (approximately on 9\%) and moment of inertia (on $20 \%$ ).

Results which are shown in Table 4, concerned mode (67), which correspond to ellipsoidal structures. The distribution of dimensionless density at maximal value of angular velocity $\Omega_{\max }$ for several values 
of angle $\theta$ is shown in Fig. 9. Built according to Table 4 the meridional section of the polytrope surface at $\Omega=\Omega_{\max }$ is shown in Fig. 11 (solid curve 2). Curve 1 corresponds to the surface of corresponding auxiliary rotational ellipsoid (with the same polar and equatorial radii, as the curve 2).

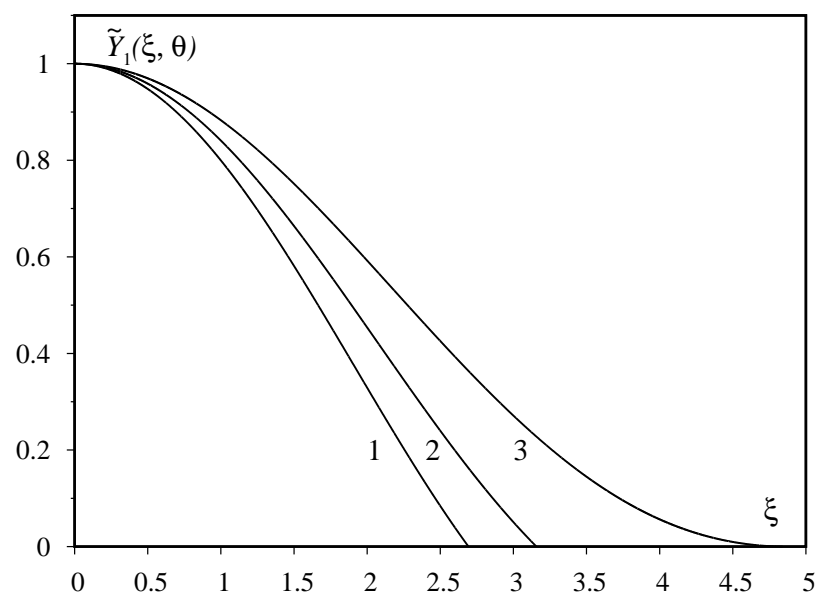

Fig. 9. The distribution of dimensionless density at $\Omega=\Omega_{\max }$ for several values of angle $\theta$ according to formula (64) in approximation $a_{2 l}=0$ at $l \geqslant 3$. Curve 1 corresponds to $\theta=0^{\circ}$, curve 2 : $\theta=45^{\circ}$, curve 3 : $\theta=90^{\circ}$.

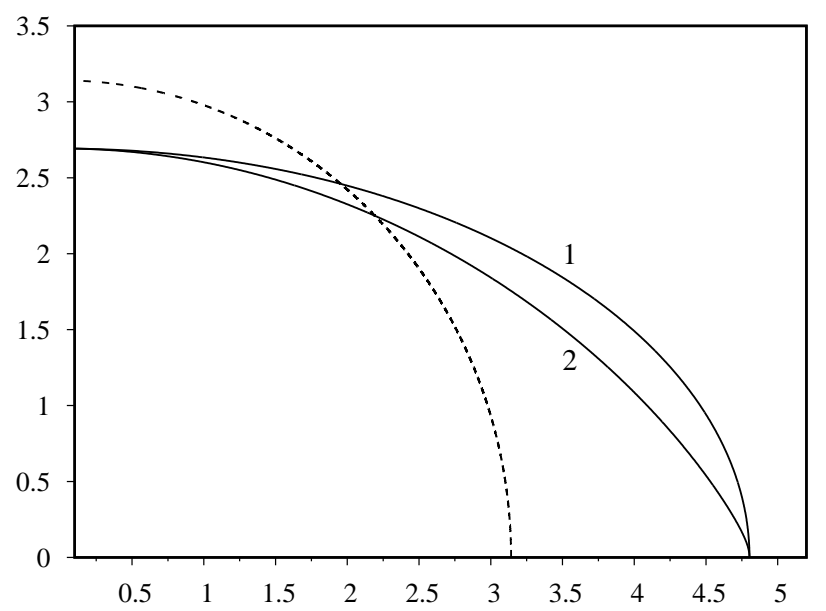

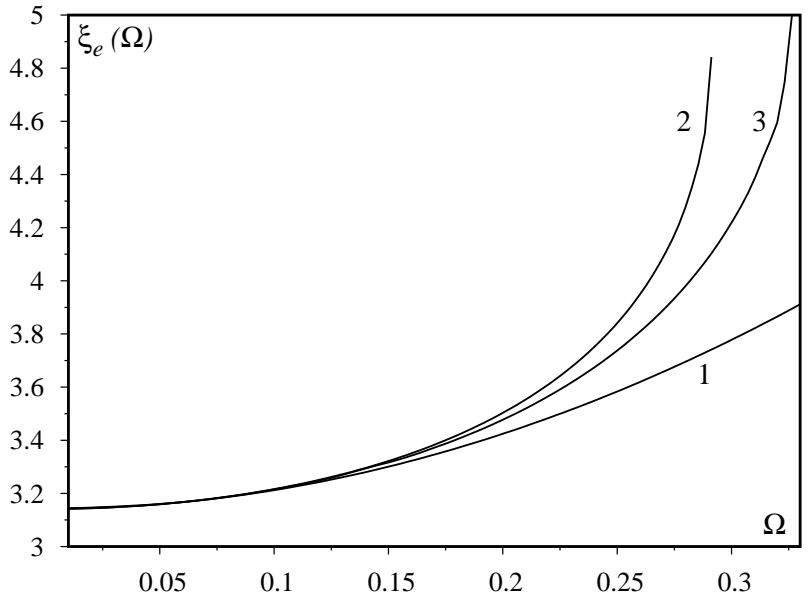

Fig. 10. Dependence of equatorial radius $\xi_{e}(\Omega)$ on angular velocity $\Omega$ for polytrope with $n=1$ in different approximations. Curve 1 is built according to results of works [7,8], curve 2 corresponds to our results. Curve 3 is built according to results of work [13].

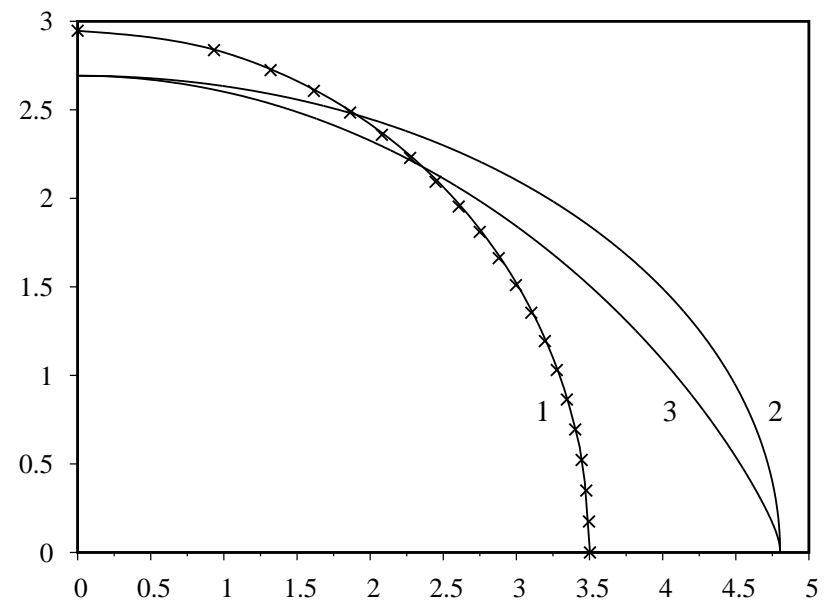

Fig. 11. The meridional section of the polytrope sur- Fig. 12. The meridional section of the polytropes face with index $n=1$ at $\Omega=\Omega_{\max }$. Curve 1 is auxil- surface with index $n=1$ taking into account $L_{4}$ at iary and corresponds to formula (40), which determines $I_{4}=0$. Curve 1 corresponds to formula (40), which the surface of ideal rotational ellipsoid, which has two determines the surface of rotational ideal ellipsoid at common points with the polytrope surface. Curve 2 is $\Omega_{1}=0.2$. Curve 2 : the same, but angular velocity built according to formula (64). Dashed curve is the $\Omega_{2}=0.2911$. Curve 3 is built according to formula (64) polytrope section without rotation. at $\Omega_{2}=0.2911$. Crosses correspond to formula (64) at

$$
\Omega_{1}=0.2 \text {. }
$$

In Fig. 10 is shown dependence of equatorial radius on angular velocity in different approximations. Curve 1 corresponds to the Milne-Chandrasekhar approximation, curve 2 is built according to Table 4 , curve 3 are results of work [13], which differs from result [8] by numerical calculation of the constant $a_{2}\left(a_{2 l}(\Omega)=0\right.$ at $\left.l \geqslant 2\right)$. Curve 2 practically coincides with the results of work [9], so we do not cite it.

\section{More accurate calculation of constants $a_{2 l}$ near $\Omega_{\max }(1)$}

Above the polytrope characteristics $n=1$ obtained in the assumption, that its surface is the surface of rotational ellipsoid, parameters of which $\xi_{e}(\Omega)$ and $e(\Omega)$ calculated by self-consistent iterative method. 
As is shown in Fig. 12, such assumption is valid in large region angular velocity change, but near the maximal $\Omega_{\max }(1)$ it disturbed, and the polytrope surface deviates from the ellipsoid surface. It follows, that in this region we should continue the iterative process, using as a starting approximation the expression $\xi_{0}(t \mid \Omega)$, which corresponds to the curve 3,

$$
\xi_{0}(t \mid \Omega)=\xi_{0}^{(0)}(t \mid \Omega)-f_{1}(t \mid \Omega)
$$

where $\xi_{0}^{(0)}(t \mid \Omega)$ is determined by formula $(40)$, and $f_{1}(t \mid \Omega)$ is deviations from the ellipsoid surface. According to definition (23)

$$
I_{2 l}=I_{2 l}(\Omega)=\left[\xi_{e}^{(0)}(\Omega)\right]^{1-2 l} \int_{0}^{1} P_{2 l}(t) f_{1}(t \mid \Omega)\left\{1+\frac{e^{2}}{1-e^{2}} t^{2}\right\}^{l-1 / 2} d t+\ldots
$$

is nonzero for all $l \geqslant 2$,

$$
\begin{aligned}
I_{2}= & I_{2}(\Omega)=\int_{0}^{1} P_{2}(t) \ln \left\{1+\frac{e^{2}}{1-e^{2}} t^{2}\right\} d t \\
& -2\left[\xi_{e}^{(0)}(\Omega)\right]^{-1} \int_{0}^{1} P_{2}(t) f_{1}(t \mid \Omega)\left\{1+\frac{e^{2}}{1-e^{2}} t^{2}\right\}^{1 / 2} d t+\ldots
\end{aligned}
$$

At calculation of matrix elements $S_{2 l, 2 l}, S_{2 m, 2 l}$ in formulae (78), (79) we have to replace $\xi_{0} \rightarrow \xi_{0}(t \mid \Omega)$. The similar replacement should be performed in formula (72) at calculation $L_{2 l}$.

The results of calculation of constants $a_{2 l}(\Omega)$, as well as the associ-

Table 5. Dependence of the model characteristics with index $n=1$ on angular velocity according to representation (64) in approximation $a_{2 l}=0, L_{2 l}=0$ and ated ellipse, which limits the top of the polytrope surface and has 2 common points with its surface $\left(\xi_{p}(\Omega)\right.$ and $\left.\xi_{e}(\Omega)\right)$ are shown in Table 5 . In Fig. 13 is shown dependence of function $f_{1}(t \mid \Omega)$ $I_{2 l}=0$ at $l \geqslant 3$.

on cosine of polar angle $t$ at $\Omega=0.2828 \ldots\left(\Omega^{2}=0.08\right)$. This function (at $L_{4} \neq 0, I_{4} \neq 0$ ) can be represented by such Padé approximant

$$
f_{1}(t \mid \Omega)=\left\{a_{0}+a_{2} t^{2}+a_{4} t^{4}+a_{6} t^{6}\right\}\left\{b_{0}+b_{2} t^{2}+b_{4} t^{4}+b_{6} t^{6}\right\}^{-1}
$$

where

$$
\begin{array}{llll}
a_{0}=6.44749 \cdot 10^{-6}, & a_{2}=0.681324, & a_{4}=6.08271, & a_{6}=-6.76694, \\
b_{0}=0.142974, & b_{2}=3.62191, & b_{4}=17.6817, & b_{6}=18.1559 .
\end{array}
$$

As was shown in Fig. 13, the deviation value of the polytrope surface from associated ellipsoid is the higher, the more accurate calculated integration constants $a_{2 l}(\Omega)$ are.

\section{Conclusions}

The solutions of differential equilibrium equation for the polytrope model with axial rotation usually are represented in the form of expansions for the Legendre polynomials, then the problem of calculation of integration constants arises (1 constant in works $[7,8], \ldots, 4$ constants in work [11]). For this purpose traditionally one has used the condition of continuity of gravitational potential on the stellar surface. At this, the gravitational potential outside the star is written 


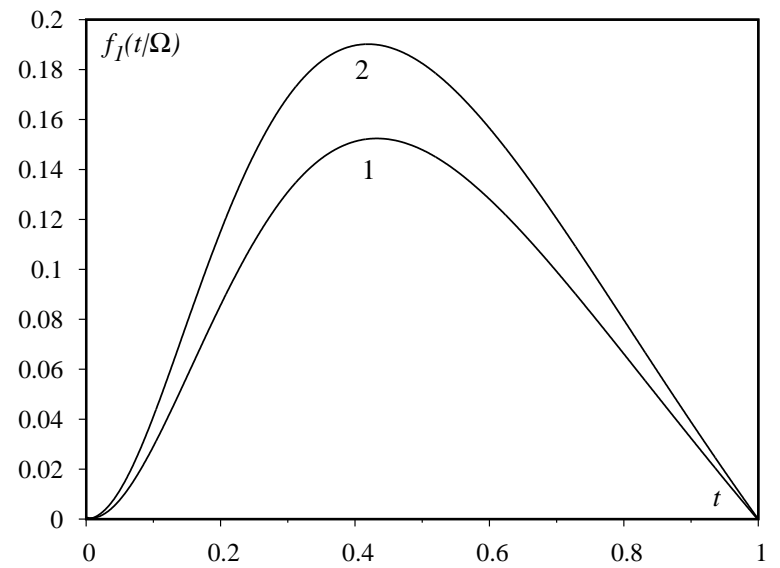

Fig. 13. Dependence of function $f_{1}(t \mid \Omega)$ on cosine of polar angle $t$ at $\Omega=0.2828 \ldots$ in different approximations. Curve 1 corresponds to approximation $L_{4} \neq 0$ at $I_{4}=0$ (according to Table 4 ), curve 2: approximation $L_{4} \neq 0$ at $I_{4} \neq 0$ (according to Table 5 ).

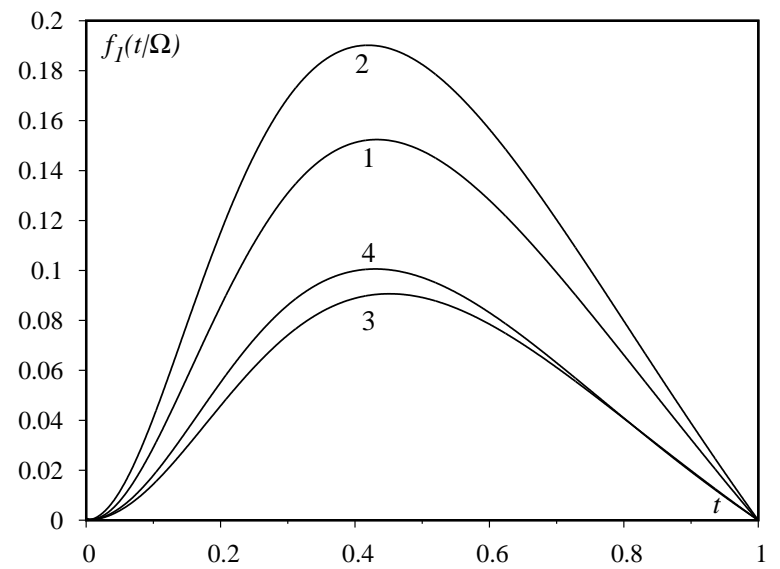

Fig. 14. Dependence of function $f_{1}(t \mid \Omega)$ on cosine of polar angle $t$ at $\Omega=0.2828 \ldots$ in different approximations. Curves 1, 2 are our results, curves 3, 4 are results of work [11]. in the form

$$
\sum_{l \geqslant 0} B_{2 l} \xi^{-1-2 l} P_{2 l}(t)
$$

but calculation of coefficients $B_{2 l}$ are not expected. Although equation (25) is relation between the solution $Y_{n}(\xi, \theta)$ and created by it gravitational potential in each point (and on its surface), this relation is self-consistent. Our approach yields an opportunity for detailed consideration of density distribution also in the vicinity of the surface at calculation of integration constants.

In the case of models $n=0$ and $n=1$, the equilibrium equation is linear, which allows us to combine analytical calculations with numerical. Model $n=0$ corresponds to the object with constant density and the problem reduces to study the polytrope surface, which determines integral equation (25). We have approved that the polytrope surface with $n=0$ is the surface of rotational ellipsoid. Obtained by us the values of polar and equatorial radii as functions of angular velocity are very close to results of work [13].

For the polytrope $n=1$ we obtained the system of linear algebraic inhomogeneous equations for calculation of integration constants with consistent consideration of multicomponent terms, which are proportional to $P_{2 l}(t)$ at $l \geqslant 1$ as in expression (64), and in equations (77). Numerical calculation were performed in approximation $P_{2 l}(t)=0$ for $l \geqslant 3$. The polytrope characteristics as functions of angular velocity $\Omega$ were calculated. It was shown that the polytrope surface at arbitrary $\Omega$ can be represented as the function of the surface of the associated rotational ellipsoid (which has common with the polytrope surface the polar and equatorial radii) and some correction $f_{1}(t \mid \Omega)$, which depends on the cosine of polar angle $t$ and angular velocity $\Omega$. In works $[8,9,11,12]$ are given only dependences of polar and equatorial radii calculated in different approximations, and the polytrope is considered as an inhomogeneous rotational ellipsoid. In works $[19,20]$ are performed calculation of star $\alpha$ Eri in the frame of polytropic model $n=1$ numerically at the value of angular velocity, which is close to observed. As can be seen in the figures given there, the polytrope surface in the vicinity of the equator has behavior, which is similar to obtained by us at large values $\Omega$.

Table 6. Dependence of polar and equatorial radii of polytrope $n=1$ on square angular velocity $\Omega^{2}$ in different approximations.

\begin{tabular}{|c|c|c|c|c|c|c|}
\hline$\Omega^{2}$ & $\xi_{p}(\Omega)$ & $\xi_{e}(\Omega)$ & $\xi_{p}(\Omega)[9]$ & $\xi_{e}(\Omega)[9]$ & $\xi_{p}(\Omega)[11]$ & $\xi_{e}(\Omega)[11]$ \\
\hline \hline 0.02 & 3.04597 & 3.29936 & 3.04590 & 3.29940 & 3.04589 & 3.29834 \\
\hline 0.04 & 2.94687 & 3.50320 & 2.94610 & 3.50320 & 2.94615 & 3.49750 \\
\hline 0.06 & 2.84175 & 3.79676 & 2.83970 & 3.79480 & 2.83952 & 3.77439 \\
\hline 0.08 & 2.72087 & 4.42030 & 2.71940 & 4.37970 & 2.71751 & 4.27332 \\
\hline
\end{tabular}

In this regard, it is worth noting work [11]. Following the work [10], the solution of the mechanical equilibrium equation in work [11] is represented in form (64), and in practical calculations taking into account terms with accuracy to $P_{8}(t)$ including. Although for calculation of integration 
constants in this work the author used traditional idea about continuity of gravitational potential on the polytrope surface, author also calculated constants $B_{2 l}$ in expansion (90), considering it suitable for the polytrope surface, although it is known that such expansion coincides absolutely only outside sphere with $\xi_{e}$ (see [25]). Author calculated values $\xi_{p}(\Omega), \xi_{e}(\Omega)$ for the same values of squared dimensionless angular velocity, as in work [9]: $\Omega^{2} \equiv 0.02,0.04,0.06,0.08$. Obtained results are very close to ones of work [9]: at $\Omega^{2}=0.08$ the deviation is $2.43 \%$ (see Table 6). However, the author of work [11] did not study the surface shape $n=1$. Using obtained by him values of integration constants, we calculated function $f_{1}(t \mid \Omega)$ of polytrope $n=1$ at $\Omega=0.2828 \ldots\left(\Omega^{2}=0.08\right)$, as is shown in Fig. 14 . Here, the curve 3 is built in approximation $a_{2 l}=0$ for $l \geqslant 3$, and the curve 4 is built in approximation $a_{2 l}=0$ for $l \geqslant 5$. Although the deviation of the polytrope surface $f_{1}^{W}(t \mid \Omega)$ on the surface of associated ellipsoid according to work [11] is slightly less than in our calculations, we observe similar regularity: function $f_{1}^{W}(t \mid \Omega)$ is the higher, the more accurate the solution of the equilibrium equation is. The maximal value $f_{1}^{\max }(\Omega)=0.5 a_{4} \Omega^{2}$.

Our analysis allows us to say, that the polytrope of rotational ellipsoid $n=1$ deviates from the surface of the associated ellipsoid. The reliability of this conclusion is ensured by the use of two different methods of calculation of integration constants, which determine the solution of the equilibrium equation. Moreover, this explains the deviation of the curves 1,2 and 3,4 : integration constant $a_{4}(\Omega)$ in work [11] at $\Omega^{2}=0.08$ are almost 2 times less than in Table 5 .

[1] McNally D. The distribution of angular momentum among main sequence stars. The Observatory. 85, 166-169 (1965).

[2] Smith F. G. Pulsars. Cambridge University Press (1977).

[3] Lane H. On the Theoretical Temperature of the Sun under the Hypothesis of a gaseous mass Maintining Its Volume by Its Internal Heat and Depending on the Law of Gases Known to Terrestrial Experiment. American Journal of Science. s2-50 (148), 57-74 (1870).

[4] Emden R. Gaskugeln: Anwendungen der mechanischen Wärmetheorie auf kosmologische und meteorologische Probleme. Leipzig, Berlin (1907), (in German).

[5] Fowler R. H. Emden's equation: The solutions of Emden's and similar differential equations. MNRAS. 91 (1), 63-91 (1930).

[6] Eddington A. S. The Internal Constitution of the Stars. Cambridge University Press (1926).

[7] Milne E. A. The equilibrium of a rotating star. MNRAS. 83 (3), 118-147 (1923).

[8] Chandrasekhar S. The Equilibrium of Distorted Polytropes. I. The Rotational Problem. MNRAS. 93 (5), 390-406 (1933).

[9] James R. A. The Structure and Stability of Rotating Gas Masses. Astrophysical Journal. 140, 552-582 (1964).

[10] Kopal Z. Bemerkung zur Theorie der rotierenden Polytropen. Zeitschrift für Astrophysik 14, 135-138 (1937), (in German).

[11] Williams P. S. Analytical Solutions for the Rotating Polytrope $N=1$. Astrophysics and Space Science. 143, 349-358 (1988).

[12] Monaghan J. J., Roxburgh I. W. The Structure of Rapidly Rotating Polytropes. MNRAS. 131 (1), 13-22 (1965).

[13] Caimmi R. Emden-Chandrasekhar Axisymmetric Solid-Body Rotating Polytropes. Part One. Exact Solutions for the Special Cases $N=0,1$ and 5. Astrophysics and Space Science. 71, 415-457 (1980).

[14] Vavrukh M. V., Tyshko N. L., Dzikovskyi D. V., Stelmakh O. M. The self-consistent description of stellar equilibrium with axial rotation. Mathematical Modeling and Computing. 6 (2), 153-172 (2019).

[15] Vavrukh M. V., Tyshko N. L., Dzikovskyi D. V. New approach in the theory of stellar equilibrium with axial rotation. Journal of Physical Studies. 24 (3), 3902-1-3902-20 (2020).

[16] Vavrukh M. V., Dzikovskyi D. V. Exact solution for the rotating polytropes with index unity, its approximations and some applications. Contrib. Astron. Obs. Skalnaté Pleso. 50 (4), 748-771 (2020). 
[17] Vavrukh M. V., Smerechynskyi S. V., Tyshko N. L. The microscopic parameters and the macroscopic characteristics of real degenerate dwarfs. Journal of Physical Studies. 14 (4), 4901-1-4901-16 (2010).

[18] Vavrukh M. V., Dzikovskyi D. V., Smerechynskyi S. V. Consideration of the competing factors in calculations of the characteristics of non-magnetic degenerate dwarfs. Ukr. J. Phys. 63 (9), 777-789 (2018).

[19] Kong D., Zhang K., Schubert G. An exact solution for arbitrarily rotating gaseous polytropes with index unity. MNRAS. 448 (1), 456-463 (2015).

[20] Knopik J., Mach P., Odrzywołek A. The shape of a rapidly rotating polytrope with index unity. MNRAS. 467 (4), 4965-4969 (2017).

[21] Shapiro S. L., Teukolsky S. A. Black Holes, White Dwarfs and Neutron Stars. Cornell University, Ithaca, New York (1983).

[22] Chandrasekhar S. An Introduction to the Study of Stellar Structure. University of Chicago Press, Chicago (1939).

[23] Lyttleton R. A. The Stability of Rotating Liquid Masses. Cambridge University Press, Cambridge (1953).

[24] Abramowitz M., Stegun I. A. Handbook of Mathematical Functions With Formulas, Graphs, and Mathematical Tables. Government Printing Office Washington (1972).

[25] Duboshin G. N. Celestial Mechanics, Basic Problems and Methods. Nauka, Moscow (1968).

\title{
Метод інтегральних рівнянь у політропній теорії зір з осьовим обертанням. І. Політропи $n=0$ i $n=1$
}

\author{
Ваврух М. В., Дзіковський Д. В. \\ Львівсъкий начіональний університет імені Івана Франка, \\ вул. Кирила і Мефодія, 8, 79005, Лъвів, Україна
}

\begin{abstract}
Розрахунки характеристик зір з осьовим обертанням у рамках політропної моделі грунтуються на розв'язку рівняння рівноваги - диференціального рівняння другого порядку в частинних похідних. Різні варіанти наближеного визначення сталих інтегрування засновані на традиційному в теорії зоряної поверхні наближенні, а саме: умові неперервності гравітаційного потенціалу в околі поверхні. Нами запропоновано новий підхід, в якому одночасно використовуються диференціальна та інтегральна форми рівняння рівноваги. Ця замкнута система дозволяє самоузгоджено визначити сталі інтегрування, форму поверхні політропи та розподіл речовини за об'ємом зорі. На прикладі політропи $n=0$ і $n=1$ встановлено існування двох режимів обертання (з малими та великими ексцентриситетами). У випадку $n=0$ доведено, що поверхня політропи є поверхнею однорідного еліпсоїда обертання. Розраховано характеристики політропи $n=1$ у різних наближеннях як функції кутової швидкості. Вперше розраховано відхилення поверхні політропи при заданому значенні кутової швидкості від поверхні асоційованого еліпсоїда обертання.
\end{abstract}

Ключові слова: зорі-політропи, неоднорідні еліпсоїди, осъове обертання, рівняння механічної рівноваги, стабільність зір. 